\title{
Long Intervening Noncoding 00467 RNA Contributes to Tumorigenesis by Acting as a Competing Endogenous RNA against miR-107 in Cervical Cancer Cells
}

\author{
Guang-Cai Li, ${ }^{*}$ Li Xin, ${ }^{\dagger}$ Yong-Sheng Wang, ${ }^{*}$ and Ying Chen* \\ From the Department of Obstetrics and Gynecology, * Linyi People's Hospital, Linyi; and the Sense Control Office, ${ }^{\dagger}$ Economic and Technological \\ Development Zone, People's Hospital of Linyi, Linyi, People's Republic of China
}

\author{
Accepted for publication \\ July 9, 2019. \\ Address correspondence to \\ Ying Chen, M.D., Department \\ of Obstetrics and Gynecology, \\ Linyi People's Hospital, No. \\ 27, Jiefang E Rd, Linyi 276003, \\ Shandong Province, People's \\ Republic of China. E-mail: dr \\ cying@yeah.net.
}

\begin{abstract}
The functional roles of individual large intervening noncoding RNAs in carcinogenesis and progression of cervical cancer have been uncovered in previous studies. In this study, we aimed to identify the role of long intervening noncoding 00467 (LINC00467) in epithelial-mesenchymal transition (EMT), invasion and migration of cervical cancer cells by regulating miR-107 and kinesin family member 23 (KIF23). Microarray analyses were used to detect cervical cancer-related differentially expressed genes, followed by determination of LINC00467, miR-107, and KIF23 levels and subcellular location of LINC00467. Cervical cancer cells were treated with a series of siRNA and mimics to measure the regulatory role of LINC00467, miR-107, and KIF23 in EMT, cell invasion, migration and proliferation, and tumorigenic ability in vivo and in vitro. LINC00467 and KIF23 were highly expressed, whereas miR-107 was poorly expressed, in cervical cancer. LINC00467 was found to be primarily located in the cytoplasm and function as a competing endogenous RNA against miR-107 to suppress KIF23. Cell proliferation, migration, invasion, and EMT in vitro were inhibited as a result of lentiviral-mediated LINC00467 knockdown and miR-107 overexpression in cervical cancer. In addition, LINC00467 silencing or miR-107 up-regulation repressed tumorigenic ability in xenograft tumor-bearing nude mice in cervical cancer in vivo. LINC00467 silencing or miR-107 up-regulation may serve as novel potential strategies for the treatment of cervical cancer. (Am J Pathol 2019, 189: 2293-2310; https://doi.org/10.1016/ j.ajpath.2019.07.012)
\end{abstract}

Cervical cancer remains to be a heavy economic burden, especially in the developing world. ${ }^{1}$ It is the second most common cancer and the third leading cause of death among women, affecting half a million women, with a mortality rate of $52 \%$ in the developing world. ${ }^{2}$ Lifestyle choices, including smoking and multiple sexual partners, are common risk factors of cervical cancer, although human papillomavirus (HPV) infection is known to be a direct cause for this debilitating disease. ${ }^{3}$ Recently, with advances in HPV vaccination and DNA examination for high-risk HPV and cytologic screening, the incidence rate of cervical cancer has been lowered. ${ }^{4}$ Although radiotherapy and radical hysterectomy have made excellent progress in treating patients with early invasive cervical cancer, patients with locally recurrent risk nevertheless experience unsatisfactory prognosis. ${ }^{5}$ Recently, long noncoding RNAs (lncRNAs) have been demonstrated to play an important part in the development and progression of cervical cancer. ${ }^{6}$ Therefore, it is reasonable to explore the effect of lncRNAs on epithelialmesenchymal transition (EMT), invasion, and metastasis in cervical cancer cells.

LncRNAs are related to DNA-binding proteins that epigenetically modulate a variety of gene expressions, and their transcription plays a vital role in gene activity during DNA damage and external oncogenic stimuli. ${ }^{7}$ In addition,

Disclosures: None declared. 
alternation of lncRNAs and associated mRNAs has been found to be related to HPV-induced cell proliferation, suggesting that lncRNAs may be involved in HPV-caused oncogenesis in cervical cancer. ${ }^{8}$ Thereby, the current study aimed to explore the effect of long intervening noncoding 00467 (LINC00467) on cervical cancer. LINC00467, an oncogenic lncRNA, is known to strengthen neuroblastoma cell survival by repressing the expression of tumor suppressor dickkopf-related protein 1, indicating that it may play a vital role in tumor formation. ${ }^{9}$

Moreover, according to our findings, LINC00467 served as a competing endogenous RNA (ceRNA) against miR-107 to regulate kinesin family member 23 (KIF23). It is associated with the formation of global regulatory networks that mediate vital biological processes. The regulatory axis of ceRNA relies on miRNA-binding sites on $3^{\prime}$ untranslated regions. ${ }^{10}$ miR-107 is a newly discovered miRNA with aberrant expression in various malignant tumors. ${ }^{11} \mathrm{~A}$ previous study showed that mithramycin A-induced miR-107 restoration, through activating p53, might provide a potential therapeutic target for the treatment of cervical cancer. ${ }^{12}$ KIF23 is a nuclear protein that regulates cellular cytokinesis. ${ }^{13}$ Overexpressed KIF23 contributes to poor patient survival in several cancers, such as breast cancer and hepatocellular carcinomas. ${ }^{14,15}$ In this study, we hypothesized that LINC00467 silencing would inhibit EMT, proliferation, migration, and invasion in cervical cancer cells by functioning as a ceRNA against miR-107 to regulate the expression of KIF23. Therefore, LINC00467 overexpression may serve as a promising biomarker for early diagnosis and prognosis of cervical cancer and silencing LINC00467 could be a therapeutic strategy.

\section{Materials and Methods}

\section{Microarray-Based Gene Expression Analysis}

Microarrays related to cervical cancer were downloaded from the Gene Expression Omnibus (https://www.ncbi.nlm.nih.gov/ geo; accession numbers GSE7803, GSE9750, and GSE63514). The Affy installation package of the $R$ language was used for standardized pretreatment of microarray data, ${ }^{16}$ and limma package was used for screening the differentially expressed genes. ${ }^{17}$ After correction, the $P$ value was expressed by adj.P.Val, and genes conforming to $\mid \log 2$ fold change $\mid>1$. 5 and adj.P.Val $<0.05$ were considered to be differentially expressed. Subsequently, heat maps of the obtained differential gene expression patterns were plotted.

\section{Study Subjects}

Patients with cervical cancer who underwent surgery at the Department of Gynecology and Obstetrics of Linyi People's Hospital (Linyi, China) from September 2015 to September 2017 were enrolled. After exclusion of patients with other malignant tumors and pregnant or lactating patients, a total of 54 patients, with a mean age of $43.94 \pm 10.54$ years (range, 26 to 76 years), were included in the current study. There were 25 patients with tumor diameter $\leq 4 \mathrm{~cm}$ and 29 patients with tumor diameter $>4 \mathrm{~cm}$. According to the International Federation of Gynecology and Obstetrics clinical staging standards of $2009,{ }^{18}$ the included 54 patients were staged as follows: stage T1a, $n=22$; stage T1b, $n=3$; stage T2a, $n=13$; and stage $\mathrm{T} 2 \mathrm{~b}, n=16$. In addition, there were 28 cases of low differentiation, 13 cases of moderate differentiation, and 13 cases of high differentiation. Cervical cancer tissues and adjacent normal tissues ( $>5 \mathrm{~cm}$ from the edge of the tumor) were collected during surgery, and resected specimens were immediately preserved in liquid nitrogen. All specimens were pathologically confirmed as cervical cancer, and none of the patients underwent radiotherapy or chemotherapy before the operation. The current study was approved by the ethics committee in Linyi People's Hospital, and signed informed consents were obtained from all participants.

\section{Real-Time Quantitative RT-PCR Data}

Total RNA was extracted from specimens using TRIzol (15596026; Invitrogen, Carlsbad, CA). Next, RNA concentration and purity were evaluated using a Nano-Drop ND-1000 spectrophotometer (Thermo Fisher Scientific, Waltham, MA). According to the instructions from the PrimeScript RT reagent kit (RR047A; Takara, Tokyo, Japan) and the TaqMan MicroRNA Reverse Transcription kit (Applied Biosystems, Inc., Foster City, CA), RNA was reverse transcribed into cDNA. Primers were designed and synthesized by Shanghai Sangon Company (Shanghai, China) (Table 1). Subsequently, a total of $20 \mu \mathrm{L}$ transcription system was applied, according to the instructions from the EasyScript First-Strand cDNA Synthesis SuperMix (AE301-02; TransGen Biotech, Beijing, China). Next, the reaction solution was used for real-time quantitative RTPCR (RT-qPCR), which was performed according to the instructions of SYBR Premix Ex Taq II kit (Takara Biotechnology Ltd, Dalian, China). RT-qPCR was performed using a fluorescence quantitative PCR (7500; Applied Biosystems, Inc.). The $2^{-\Delta \Delta C t}$ method was applied to indicate differences in the target gene expression among the experiment and control groups.

\section{Western Blot Analysis}

Tissues or cells were lysed with radioimmunoprecipitation assay (P0013B; Beyotime Biotechnology Co, Shanghai, China) lysis solution containing phenylmethylsulfonyl fluoride and phosphatase inhibitor on ice for 30 minutes. Protein concentration was detected by a bicinchoninic acid protein quantification kit (Beyotime Biotechnology Co). Subsequently, SDS-PAGE was performed with $30 \mu \mathrm{g}$ protein. Protein was then transferred to a nitrocellulose membrane and sealed with 5\% skimmed milk powder (prepared 
Table 1 RT-qPCR Primer Sequences

\begin{tabular}{ll}
\hline Target genes & Sequences \\
\hline LINC00467 & F: 5'-GGCAGGGAGGTTTAATAGAC-3' \\
miR-107 & F: 5' 5'-TACCTTCATATTTGCCAGCC-3' $^{\prime}$ \\
KIF23 & R: 5'-GACTCTACGACTACTAAATCG-3' \\
MMP2 & F: 5'-TGGTTCCTACATTCAGAAATGAGA-3' \\
& R: 5'-CGTTCTGATCAGGTTGAAAGAGTA-3' \\
MMP9 & F: 5'-CACCCATTTACACTACACC-3' \\
& R: 5'-GTTTTTGCTCCAGTTAAAGG-3' \\
U6 & F: 5'-CCCGGACCAAGGATACAG-3' \\
& R: 5'-GGCTTTCTCTCGGTACTG-3' \\
GAPDH & F: 5'-CTCGCTTCGGCAGCACA-3' \\
& F: 5'-AACGCTTCACGAATTTGCGT-3' \\
& R: 5'-GAGCGAGATCCCTCCAAAAT-3' \\
\hline
\end{tabular}

F, forward; GAPDH, glyceraldehyde-3-phosphate dehydrogenase; KIF23, kinesin family member 23; MMP, matrix metallopeptidase; R, reverse; RTqPCR, real-time quantitative RT-PCR.

with tris-buffered saline and Tween-20) for 1.5 hours. The membrane was probed with the primary antibodies, including rabbit anti-human antibody to KIF23 (ab9259; dilution 1:1000), matrix metallopeptidase (MMP) 2 (ab92536; dilution 1:1000), MMP9 (ab73734; dilution 1:1000), and glyceraldehyde-3-phosphate dehydrogenase (ab9485; dilution 1:2500) at $4^{\circ} \mathrm{C}$ overnight. After that, diluted horseradish peroxidase-labeled secondary antibody, goat anti-rabbit antibody to IgG (ab205718; dilution 1:2000 to $1: 50,000)$ was added to the membrane for incubation at room temperature for 2 hours. All aforementioned antibodies were purchased from Abcam (Cambridge, MA). Then, the membrane was developed with an enhanced chemiluminescence solution and imaged by SmartView Pro 2000 (UVCI-2100; Major Science, Saratoga, CA). The ratios of gray values of the protein bands were analyzed using the Quantity One software version 4.6.6. ${ }^{19}$

\section{Fluorescence in Situ Hybridization}

A coverslip was placed at the bottom of a 24-well plate for cell culture at a density of $6 \times 10^{4}$ cells/well. The cell confluence was allowed to reach $60 \%$ to $70 \%$ before experimentation. Cells were then fixed with $4 \%$ paraformaldehyde at room temperature for 10 minutes and reacted with $1 \mathrm{~mL}$ precooled permeation solution at $4^{\circ} \mathrm{C}$ for 5 minutes. Next, prehybridization solution $(20 \mu \mathrm{L})$ was added to each well and sealed at $37^{\circ} \mathrm{C}$ for 30 minutes. Meanwhile, the hybridization solution was preheated at $37^{\circ} \mathrm{C}$. After prehybridization solution in each well was discarded, cells were added with Stellaris RNA FISH (Biosearch Technologies, Petaluma, CA) probe hybridization solution containing a LINC00467-specific probe in dark conditions and hybridized at $37^{\circ} \mathrm{C}$ overnight. Subsequently, cells in each well were washed with lotion I, lotion II, and lotion III at $42^{\circ} \mathrm{C}$, avoiding exposure to light. Afterward, the cells were stained with DAPI for 10 minutes in dark conditions. The coverslip was then carefully removed from the well, and the slides were fixed with the mounting agent in dark conditions. Finally, the cells were observed, and fluorescence was detected under a fluorescence microscope (Olympus, Tokyo, Japan).

\section{Dual-Luciferase Reporter Gene Assay}

The target gene of miR-107 was predicted with prediction websites TargetScan version 7.1 (http://www.targetscan.org/ vert_71, last accessed December 19, 2018) and LncTBD version 2.0 (http://www.bio-bigdata.net/LncACTdb, last accessed December 19, 2018). Subsequently, a dualluciferase reporter gene assay was conducted to examine whether LINC00467 and KIF23 were the direct targets of miR-107. The wide-type (WT) target site sequence and mutant-type (MUT) sequence obtained from site-specific mutagenesis on WT of LINC00467 and the $3^{\prime}$ untranslated region of KIF23 mRNA were synthesized. pmiR-RBREPORTTM plasmid (RiboBio Company, Guangzhou, China) was enzymatically digested using restrictive endonuclease. Synthesized target gene segments (WT and MUT) were then inserted into the pmiR-RB-REPORT vectors (RiboBio Company) with miR-107 mimic. Simultaneously, empty plasmid transfection was regarded as the control group. Firefly and Renilla luciferase activities were determined at the 48-hour time interval after transfection using a luciferase detection reagent kit (RG005; Beyotime Biotechnology Co). Results were expressed as follows: the relative luciferase activity $=$ relative luciferase units of firefly luciferase/relative luciferase units of Renilla luciferase. ${ }^{20}$

\section{RIP Data}

Protein binding of LINC00467 and miR-107 was detected using an RNA immunoprecipitation (RIP) reagent kit (Millipore Inc., Bedford, MA). After detection, HeLa cells were lysed with equal volumes of radioimmunoprecipitation assay lysis solution (P0013B; Beyotime Biotechnology Co) for 5 minutes and centrifuged at $35,068 \times g$ at $4{ }^{\circ} \mathrm{C}$ for 10 minutes to obtain the supernatant. A portion of the cell extracting solution was used as the input, whereas the remaining solution was incubated with antibody for precipitation. The magnetic beads-antibody complex was cleaned, resuspended in $900 \mu \mathrm{L}$ RIP wash buffer, and incubated at $4^{\circ} \mathrm{C}$ overnight with $100 \mu \mathrm{L}$ cell extracting solution. Magnetic bead-protein complex was collected from the samples on the magnetic base. RNAs in the samples and input were separately extracted after protease K removal for subsequent PCR detection. Rabbit anti-human argonaute 2 (ab186733; dilution 1:50; Abcam, Shanghai, China) was well mixed at room temperature for 30 minutes, whereas rabbit anti-human IgG (ab109489; dilution 1:100; Abcam, Shanghai, China) was used as the negative control (NC). 

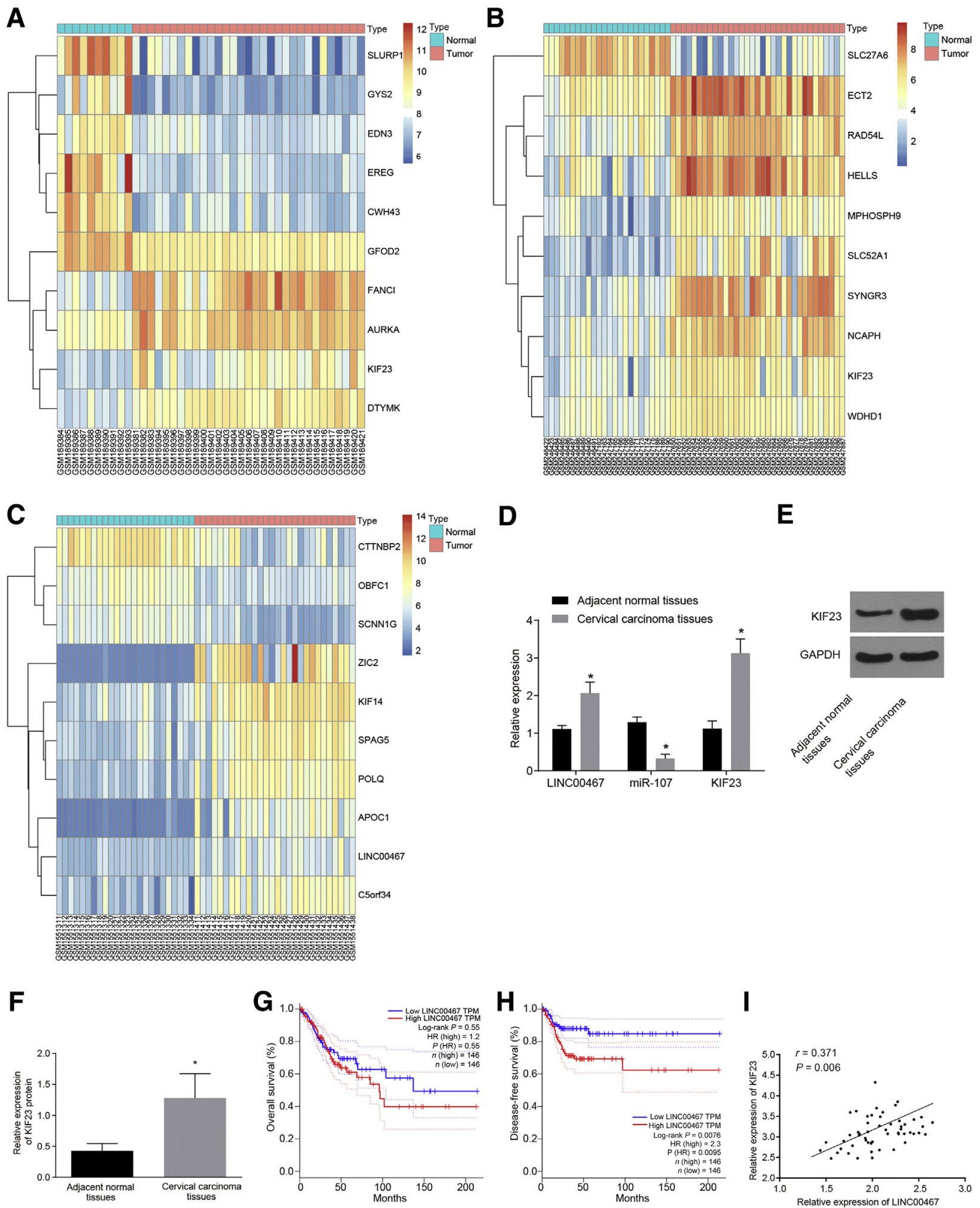

Figure 1 The expression of LINC00467 and kinesin family member 23 (KIF23) is elevated, whereas that of miR-107 is reduced, in cervical cancer tissues. A: Heat map of GSE7803 screening. B: Heat map of GSE9750 screening. C: Heat map of GSE63514 screening. D: Expression of miR-107, LINC00467, and KIF23 in cervical cancer tissues and adjacent normal tissues detected by real-time quantitative RT-PCR (RT-qPCR). E: KIF23 protein band detected by Western blot analysis. F: Relative expression of KIF23 detected by Western blot analysis. G: Overall survival curves for 54 cervical cancer patients with high or low LINC00467 expression. H: Disease-free survival curves for 54 cervical cancer patients with high or low LINC00467 expression. I: Correlation between LINC00467 and mRNA expressions of KIF23 in cervical cancer samples, and the line represents the reference line in the Pearson's analysis of LINC00467 and KIF2. The results of RTqPCR and Western blot analysis were measurement data, which were examined by unpaired $t$-test. Data are expressed as means \pm SD $(\mathbf{D}$ and $\mathbf{F}) . n=54(\mathbf{D}-\mathbf{I})$. ${ }^{*} P<0.05$ versus adjacent normal tissues. GAPDH, glyceraldehyde-3-phosphate dehydrogenase; HR, hazard ratio; TPM, transcripts per million. 
Table 2 Association of LINC00467 Expression with the Clinicopathologic Features of Patients with Cervical Cancer

\begin{tabular}{|c|c|c|c|}
\hline Clinicopathologic features & $n$ & $\begin{array}{l}\text { LINC00467 } \\
\text { relative expression }\end{array}$ & $P$ value \\
\hline Age, years & & & 0.622 \\
\hline$\leq 45$ & 32 & $2.05 \pm 0.27$ & \\
\hline$>45$ & 22 & $2.09 \pm 0.32$ & \\
\hline Tumor diameter, $\mathrm{cm}$ & & & $<0.001$ \\
\hline$\leq 4$ & 25 & $1.92 \pm 0.29$ & \\
\hline$>4$ & 29 & $2.20 \pm 0.22$ & \\
\hline Histologic grade & & & $<0.001$ \\
\hline Poorly differentiated & 28 & $2.29 \pm 0.18$ & \\
\hline Moderately differentiated & 13 & $1.87 \pm 0.14$ & \\
\hline Well differentiated & 13 & $1.78 \pm 0.18$ & \\
\hline TNM staging & & & $<0.001$ \\
\hline $\mathrm{Ia} / \mathrm{b}$ & 25 & $1.89 \pm 0.26$ & \\
\hline $\mathrm{IIa} / \mathrm{b}$ & 29 & $2.22 \pm 0.21$ & \\
\hline
\end{tabular}

TNM, tumor-node-metastasis.

\section{RNA Pull Down}

A total of $50 \mathrm{nmol} / \mathrm{L}$ biotin-labeled WT-bio-miR-107 and MUT-bio-miR-107 (Wuhan GeneCreate Biological Engineering Co, Ltd, Wuhan, China) was used to transfect HeLa cells. Cells were incubated in specific lysis buffer (Ambion, Austin, TX) for 10 minutes. The lysate was incubated in vortex with M-280 streptavidin magnetic beads (S3762; Sigma-Aldrich Chemical Company, St. Louis, MO) precoated with RNase-free bovine serum albumin and yeast tRNA (TRNABAK-RO; Sigma-Aldrich Chemical Company) at $4{ }^{\circ} \mathrm{C}$ for 3 hours. Then, beads were washed twice with precooled lysis buffer, thrice with low salt buffer, and once with high salt buffer. The combined RNA was purified with TRIzol, followed by detection of LINC00467 enrichment through RT-qPCR. ${ }^{21}$

\section{Cell Culture and Treatment}

Cervical cancer cell lines HeLa (CL-0101) and $\mathrm{SiHa}$ (CL-0210) were provided by Procell (Wuhan, China), which were exposed to RPMI 1640 medium (Gibco, Carlsbad, CA) with $10 \%$ fetal bovine serum in a $5 \% \mathrm{CO}_{2}$ incubator at $37^{\circ} \mathrm{C}$. Cells at the logarithmic phase of growth were then assigned into the following groups: experiment 1, the blank group (no transfection and treated with phosphate-buffered saline), the small interfering (si)-LINC00467 NC group (transfected with si-LINC00467 NC plasmid), the si-LINC00467 group (transfected with si-LINC00467 plasmid), the LINC00467
NC group (transfected with LINC00467 NC plasmid), and the LINC00467 group (transfected with LINC00467 plasmid). In the second phase of the experiment, the following groups were transfected: the blank group (no transfection and treated with phosphate-buffered saline), the miR-107 mimic NC group (transfected with miR-107 mimic NC plasmid), the miR-107 mimic group (transfected with miR-107 mimic plasmid), the KIF23 NC group (transfected with KIF23 NC plasmid), the KIF23 group (transfected with KIF23 plasmid), the si-NC group (transfected with si-NC plasmid), the siLINC00467 group (transfected with si-LINC00467 plasmids), the miR-107 mimic + KIF23 group (cotransfected with miR-107 mimic and KIF23 plasmids), the siLINC00467 + miR-107 mimic group (cotransfected with siLINC00467 and miR-107 mimic plasmids), and the siLINC00467 + KIF23 group (cotransfected with siLINC00467 and KIF23 plasmids). All aforementioned target plasmids were purchased from Dharmacon (Lafayette, CO).

\section{Lentivirus Package, Purification, and Transfection}

Cells from each group were seeded into 6-well plates at a density of $10^{5}$ cells/well. When cell confluence reached $80 \%$, polyethylenimine (Beijing Solarbio Science \& Technology Co, Ltd, Beijing, China) was used for transfection. Opti-minimal essential medium (750 $\mu \mathrm{L}$; Gibco) was used to dilute lentiviral vector protocadherin of $10 \mu \mathrm{g}$ target plasmids, including LINC00467, KIF23, si-LINC00467, si-KIF23, and helper plasmids, including $7.5 \mu \mathrm{g}$ paxillin and $5 \mu \mathrm{g}$ pMD2G. Next, polyethylenimine $(112.5 \mu \mathrm{g})$ was diluted with $750 \mu \mathrm{L}$ opti-minimal essential medium, mixed lightly, and then kept at room temperature for 5 minutes. After 20 minutes, the mixture was added to $10-\mathrm{cm}$ cell culture dishes pretreated with $5 \mathrm{~mL}$ Dulbecco's modified Eagle's medium for 30 minutes and cultured in a $5 \% \mathrm{CO}_{2}$ incubator at $37^{\circ} \mathrm{C}$. After incubation for a 6-hour period, the cells were further cultured in $8 \mathrm{~mL}$ complete medium for 48 hours, followed by collection of the supernatant. With $8 \mathrm{~mL}$ complete medium added, the supernatant was collected after 24 hours, centrifuged at $800 \times g$ for 5 minutes, and filtered using a $0.45-\mu \mathrm{m}$ filter membrane. Then, $30 \mathrm{~mL}$ filtrate and $7.5 \mathrm{~mL}$ of $5 \times$ polyethylene glycol 8000 solution (8.776 g NaCl and $50 \mathrm{~g}$ polyethylene glycol 8000) were dissolved in $200 \mathrm{~mL}$ ultrapure water, sterilized at $121^{\circ} \mathrm{C}$ for 30 minutes, and preserved at $4^{\circ} \mathrm{C}$. The solution was mixed every 30 minutes for three to five times and allowed to stand at $4^{\circ} \mathrm{C}$ overnight. Cells were then centrifuged at $4^{\circ} \mathrm{C}$ at $4000 \times g$ for 40 minutes. Pellets were resuspended with $1 \mathrm{~mL}$ Dulbecco's
LINC00467

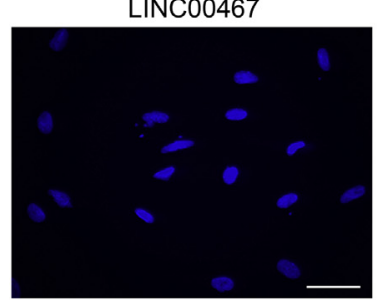

DAPI

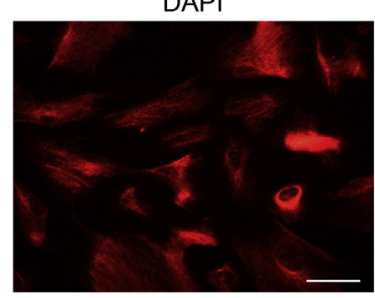

Merge

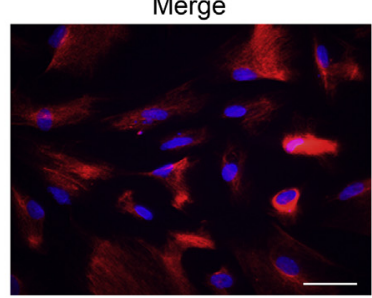

Figure 2 LINC00467 is primarily located in the cytoplasm. Scale bars $=25 \mu \mathrm{m}$. 
A
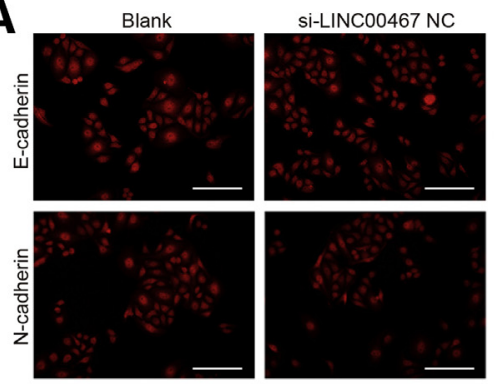

C
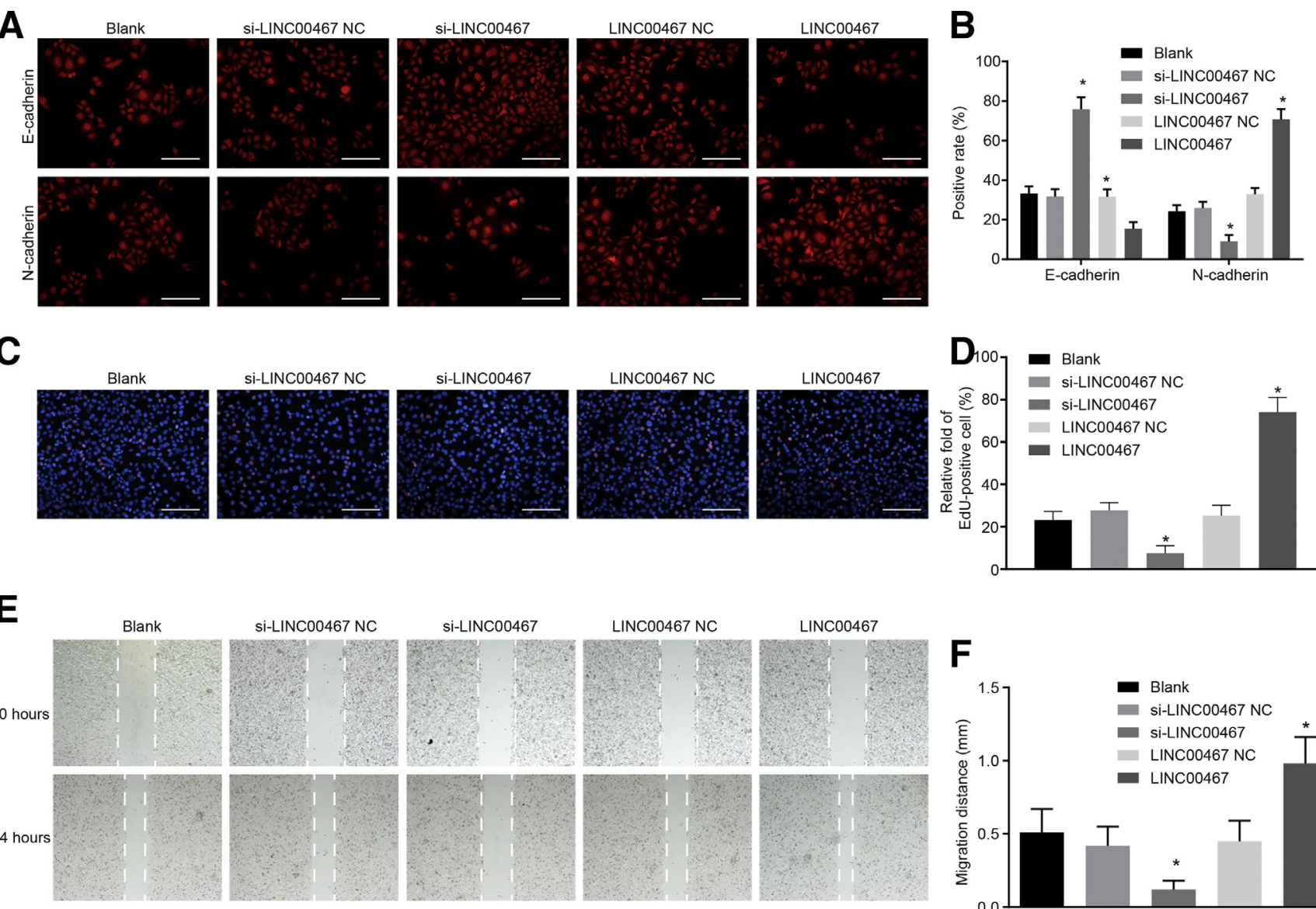

$\mathbf{F}$

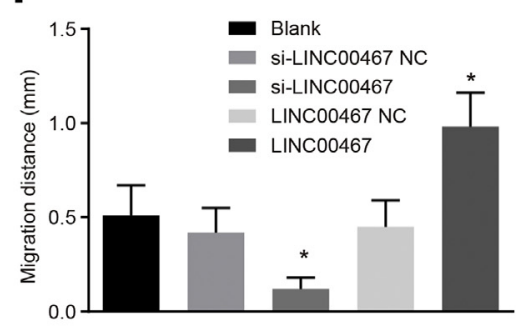

G
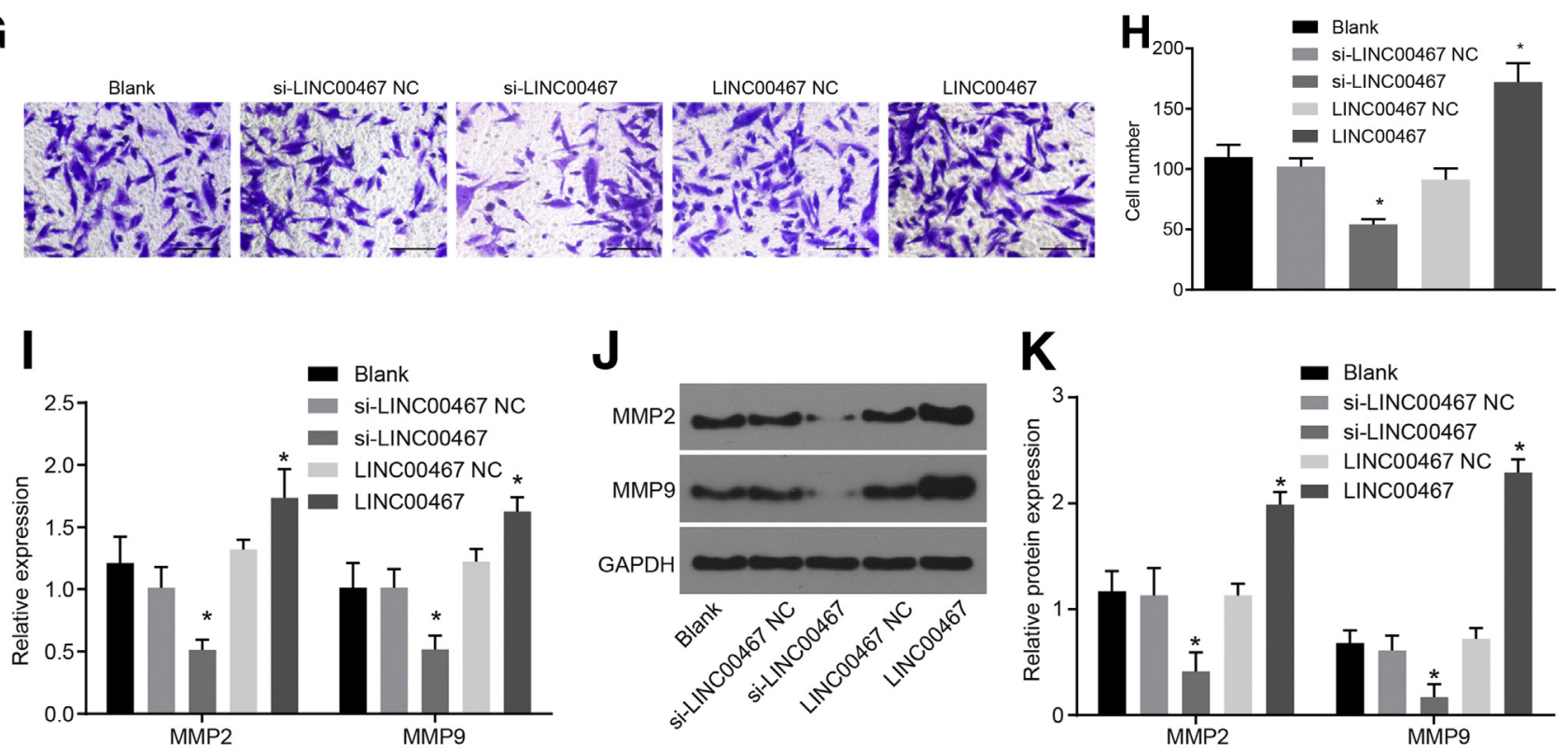

Figure 3 LINC00467 silencing delays epithelial-mesenchymal transition, proliferation, migration, and invasion in HeLa cells. A: Representative immunofluorescence images showing neural cadherin (N-cadherin) and epithelial cadherin (E-cadherin) in cervical cancer cells. B: Expression of N-cadherin and E-cadherin in cervical cancer cells. C: Representative images of cervical cancer cell proliferation detected by 5-ethynyl-2'-deoxyuridine (EdU) assay. D: Percentage of EdU-stained positive cervical cancer cells. E: Representative images of the width of scratches in cervical cancer cells detected by scratch test; the dotted lines indicate the current migration location of cells. F: Migration distance of cervical cancer cells after different treatment. G: Representative images showing cell invasion in cervical cancer cells. H: Graph showing cell numbers in transwell assay. I: Graph showing relative expression of matrix metallopeptidase (MMP) 2 and MMP9 in cervical cancer cells. J: Gray value analysis of MMP2, MMP9, and glyceraldehyde-3-phosphate dehydrogenase (GAPDH) in cervical cancer cells. K: Protein expression of MMP2 and MMP9 in cervical cancer cells. Immunofluorescence value, cell proliferation value in EdU, and migration distance in scratch test were measurement data, which were analyzed by one-way analysis of variance; the experiment was independently repeated three times. Data are expressed as means \pm SD (B, D, F, H, I, and $\mathbf{K}$ ). ${ }^{\star} P<0.05$ versus the blank group. Scale bars $=50 \mu \mathrm{m}(\mathbf{A}, \mathbf{C}$, and $\mathbf{G})$. NC, negative control. 


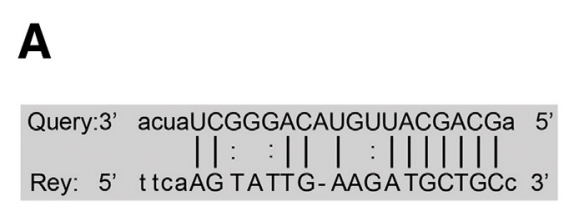

\section{B}

C

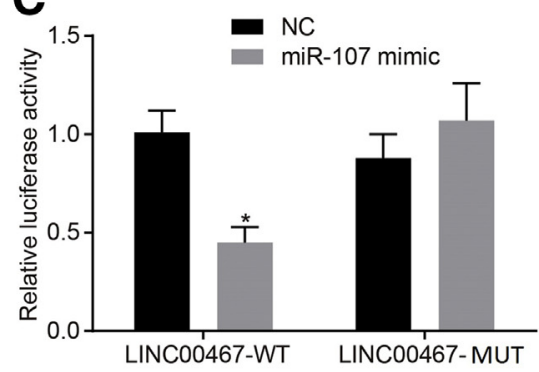

$\mathbf{F}$

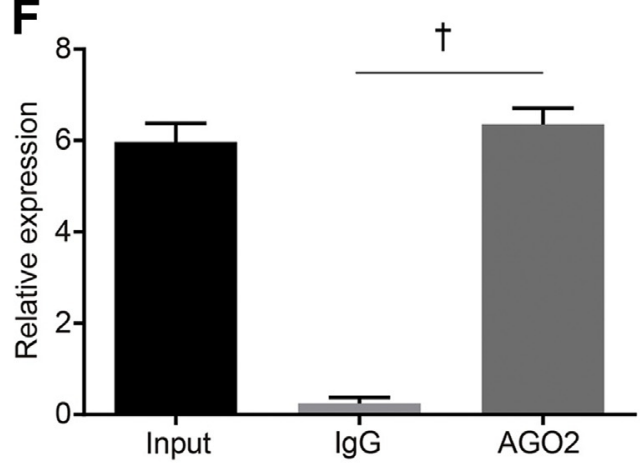

H

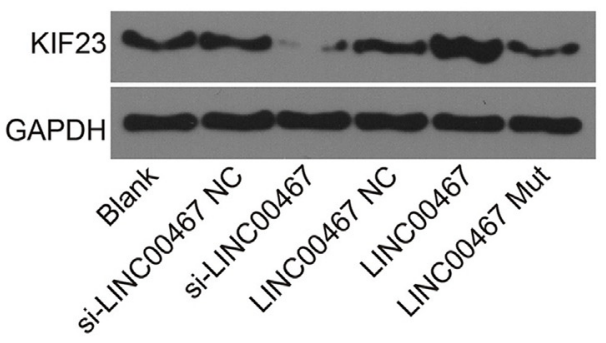

Predicted consequential pairing of target region (top) and miRNA (bottom)

\begin{tabular}{|c|c|}
\hline $\begin{array}{l}\text { Position } 430-436 \text { of KIF23 } 3^{\prime} \text { UTR } \\
\text { hsa-miR-144-3p }\end{array}$ & $\begin{array}{l}5^{\prime} \ldots \text { AGGUUUUAUAAACAAAUGCUGCU } \\
1111111 \\
3^{\prime} \\
\text { ACUAUCGGGACAUGUUACGACGA }\end{array}$ \\
\hline
\end{tabular}

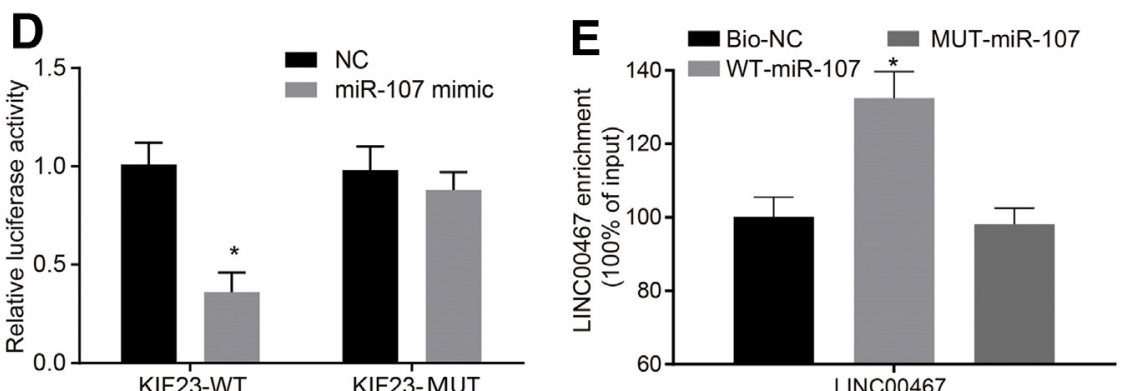

G

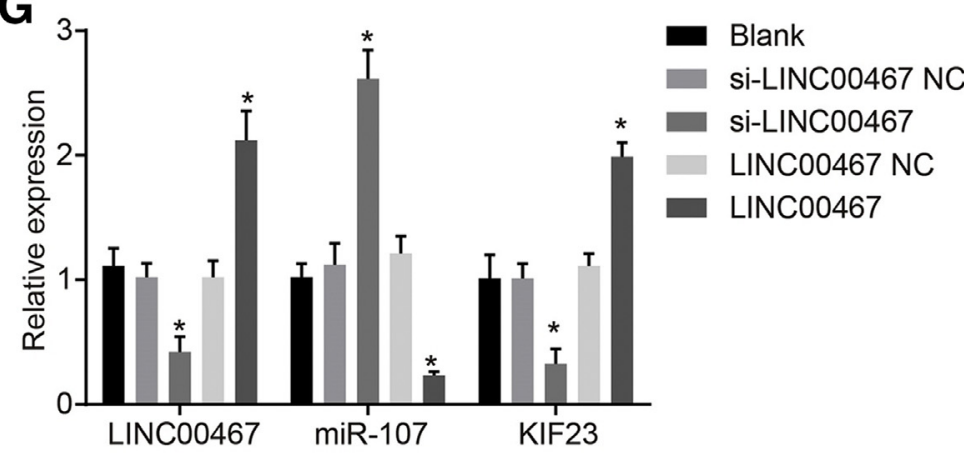

I

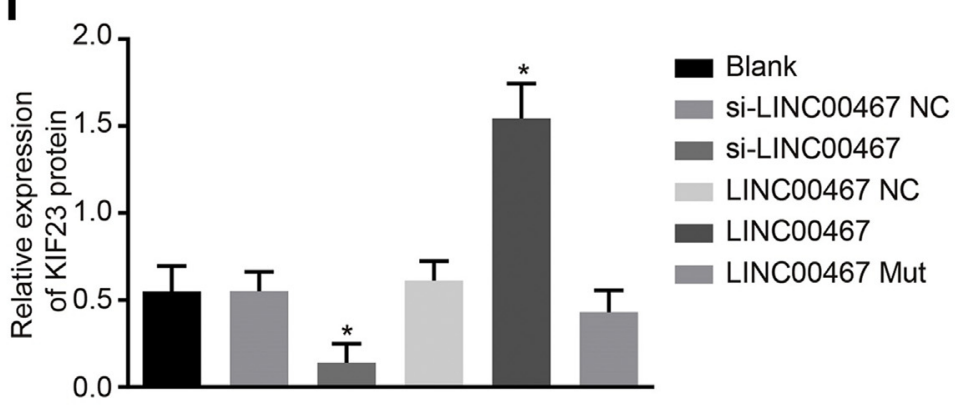

Figure 4 LINC00467 sponges miR-107 to regulate kinesin family member 23 (KIF23). A: Binding sites between miR-107 and LINC00467. B: Binding sites between miR-107 and KIF23. C: Luciferase activity of miR-107 and LINC00467. D: Luciferase activity of miR-107 and KIF23. E: RNA binding between LINC00467 and miR-107 by RNA pull-down assay. F: RNA binding between LINC00467 and argonaute 2 (AG02) detected by RNA immunoprecipitation assay. G: Relative expression of miR-107, LINC00467, and KIF23. H: Gray value analysis of KIF23 protein bands. I: Protein expression of KIF23 in each group. Luciferase activity and results of real-time quantitative RT-PCR were measurement data. Luciferase activity was examined by unpaired $t$-test, and other data were analyzed by one-way analysis of variance. The experiment was independently repeated three times. Data are expressed as means $\pm \mathrm{SD}(\mathbf{C}-\mathbf{G}$ and $\mathbf{I})$. * $P<0.05$ versus the negative control (NC) group; ${ }^{\dagger} P<0.05$ versus IgG. GAPDH, glyceraldehyde-3-phosphate dehydrogenase; MUT, mutant type; UTR, untranslated region; WT, wild type.

modified Eagle's medium and conserved at $4^{\circ} \mathrm{C}$. A day before experimentation, the well-developed 293T cells were treated, centrifuged, and then dispersed into cell suspension. The cells were then seeded into a 24 -well plate at a density of 8000 cells/ well. On experiment day, $500 \mu \mathrm{L}$ complete medium (including $0.05,0.5,5$, and $50 \mu \mathrm{L}$ lentivirus separately) was added to each well and incubated in a $5 \% \mathrm{CO}_{2}$ incubator for 72 hours. Then, 293T cells from each well were detached, followed by determination of the proportion of green fluorescent protein-positive cells using flow cytometry. Activated lentiviral particles in $1 \mathrm{~mL}$ concentrated solution were calculated using the current most common formula for the calculation of titer of viruses: virus titer (transducing units $/ \mathrm{mL}$ ) = $(\mathrm{A}+\mathrm{B} \times 10) \times 1000 / 2 / \mathrm{A}$, where $\mathrm{A}$ was the average number of fluorescent cells with the penultimate visible fluorescence well and $\mathrm{B}$ was the average number of fluorescent cells with the ultimate visible fluorescence well. HeLa cells were seeded to a 6 -well plate at a density of $3 \times 10^{5}$ cells/well. Fresh complete 

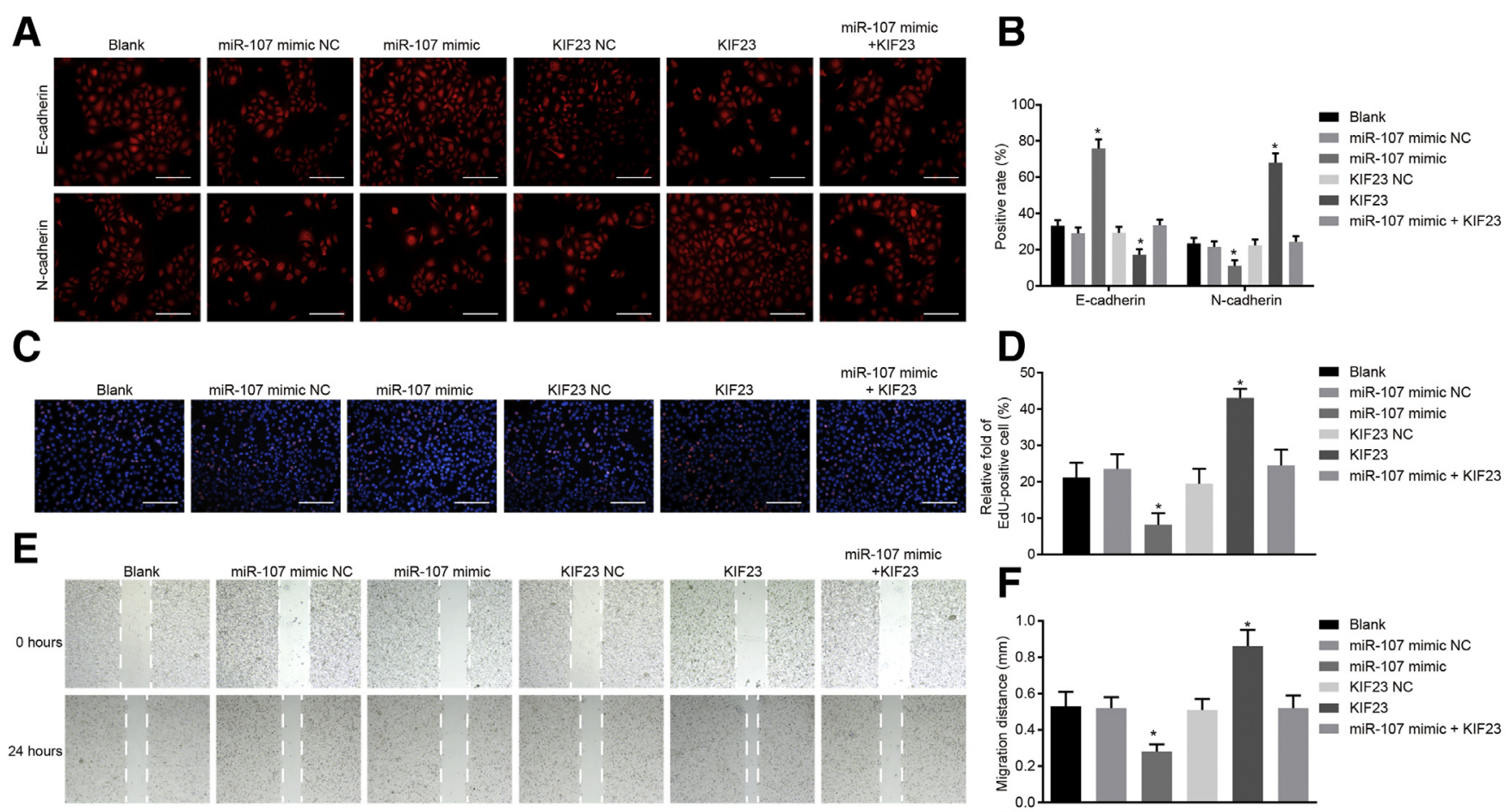

$\underset{\text { miR-107 mimic }}{+ \text { KIF23 }}$
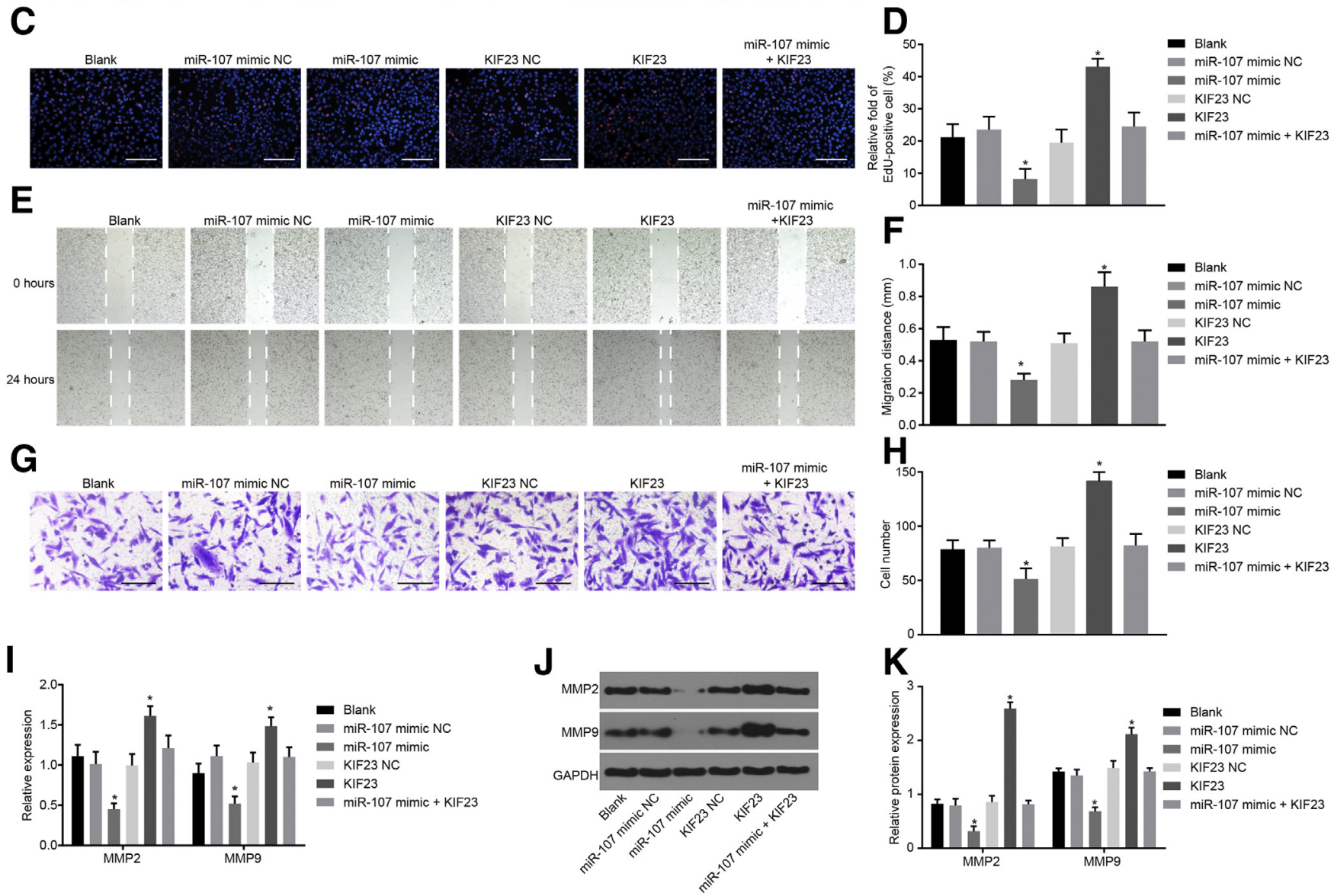

Figure 5 Elevated miR-107 restrains HeLa cell epithelial-mesenchymal transition, proliferation, migration, and invasion. A: Representative immunofluorescence images showing neural cadherin (N-cadherin) and epithelial cadherin (E-cadherin). B: Graph showing expression of $\mathrm{N}$-cadherin and $\mathrm{E}$-cadherin in cervical cancer cells. C: Representative images of cervical cancer cell proliferation detected by 5-ethynyl-2'-deoxyuridine (EdU) assay. D: Percentage of EdUstained positive cervical cancer cell. E: Representative images showing the width of scratch in cervical cancer scratch test; the dotted lines indicate the current migration location of cells. F: Migration distance in cervical cancer cells after different treatment. G: Representative images showing cell invasion in transwell assay. H: Graph showing cervical cancer cell number in transwell assay. I: Graph showing relative expression of matrix metallopeptidase (MMP) 2 and MMP9. J: Gray value analysis of MMP2, MMP9, and glyceraldehyde-3-phosphate dehydrogenase (GAPDH). K: Protein expression of MMP2 and MMP9. Immunofluorescence assay, cell proliferation value of each group in EdU assay, and migration distance in scratch test were measurement data, which were analyzed by one-way analysis of variance. The experiment was independently repeated three times. Data are expressed as means \pm SD $(\mathbf{B}, \mathbf{D}, \mathbf{F}, \mathbf{H}, \mathbf{I}$, and $\mathbf{K})$. ${ }^{*} P<0.05$ versus the blank group. Scale bars $=50 \mu \mathrm{m}(\mathbf{A}, \mathbf{C}$, and G). KIF23, kinesin family member 23; NC, negative control.

medium was added until cell confluence reached $50 \%$ to $80 \%$, and then cells were transfected using a Polybrene reagent kit (Sigma-Aldrich, St. Louis, MO).

\section{Immunofluorescence Assay}

Cells were collected with trypsin treatment. After centrifugation, sterilized coverslips $(24 \times 24 \mathrm{~mm})$ were placed on the culture dish for inoculation with different cell lines. After 24 hours, when cell confluence reached $70 \%$, the cells were allowed to grow on coverslips. Cells were then fixed with $4 \%$ formaldehyde at room temperature for 20 minutes, sealed with $10 \%$ goat serum for 15 minutes, and incubated with rabbit anti-human antibodies, epithelial cadherin (E-cadherin; ab40772; dilution 1:500), and neural cadherin (N-cadherin; ab18203; dilution $1: 5000)$ at $4^{\circ} \mathrm{C}$ overnight. Next, the cells were rewarmed in a 

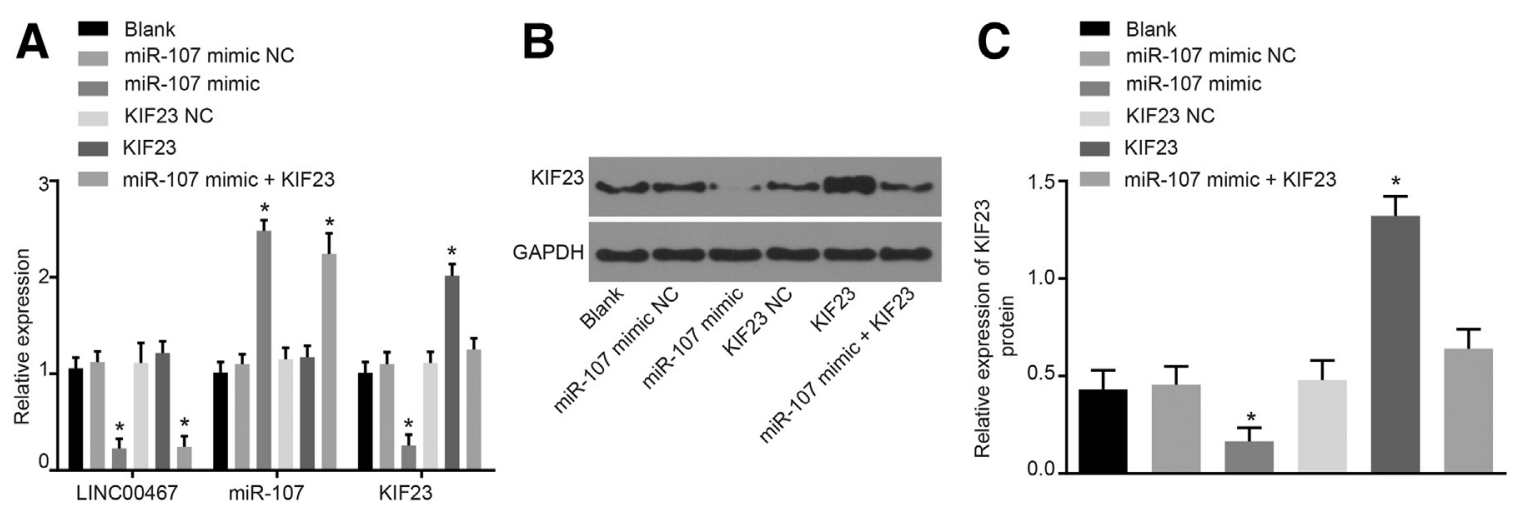

Figure 6 Silencing of LINC00467 or enhanced miR-107 reduces the expression of kinesin family member 23 (KIF23). A: Graph showing relative expression of LINC00467, miR-107, and KIF23 in cervical cancer cells after transfection. B: Gray value analysis of KIF23 in cervical cancer cells after transfection. C: Protein expression of KIF23 in cervical cancer cells after transfection. Cellular experiment data of real-time quantitative RT-PCR and Western blot analysis were included in measurement data. Comparison among multiple groups was analyzed by one-way analysis of variance, and the experiment was independently repeated three times. Data are expressed as means $\pm \mathrm{SD}(\mathbf{A}$ and $\mathbf{C}) .{ }^{*} P<0.05$ versus the blank group. GAPDH, glyceraldehyde-3-phosphate dehydrogenase; NC, negative control.

$37^{\circ} \mathrm{C}$ incubator for 45 minutes. The secondary antibody, fluorescence-labeled goat anti-rabbit IgG (ab205718; dilution 1:2000 to 50,000) was then added for a 2-hour incubation at room temperature in the dark. The aforementioned antibodies were purchased from Abcam (Cambridge, MA). After incubation, the cells were stained with DAPI (dilution 1:10; Vector Laboratories, Burlingame, CA) for 5 minutes in dark conditions, dehydrated with gradient ethanol, cleared with xylene, sealed with neutral balsam, and observed under a microscope.

\section{EdU Assay}

Cells from each group were seeded in a 96-well plate at a density of $1.6 \times 10^{5}$ cells/well and cultured for 48 hours. Subsequently, $50 \mathrm{mmol} / \mathrm{L}$ 5-ethynyl- $2^{\prime}$-deoxyuridine (EdU) was added to each well and incubated at $37^{\circ} \mathrm{C}$ for 4 hours. Cells were then fixed with $4 \%$ formaldehyde at room temperature for 15 minutes and treated with $0.5 \%$ Triton X-100 at room temperature for 20 minutes for permeabilization. Next, the cells were incubated with $100 \mathrm{~mL}$ Apollo mixture (Guangzhou RiboBio Co., Ltd., Guangzhou, Guangdong, China) at room temperature for 30 minutes, stained with $100 \mathrm{~mL}$ Hoechst33342 (Sigma-Aldrich, St Louis, MO) for 30 minutes, and imaged under a fluorescence microscope (Olympus, Tokyo, Japan). The Image-Pro Plus software version 6.0 (Media Cybernetics, Bethesda, MD) was used to calculate the number of EdU-positive cells (red cells). EdU incorporation efficiency was presented as the proportion of EdU-positive cells in HeLa-positive cells (blue cells). ${ }^{22}$

\section{Scratch Test}

Horizontal lines were scratched uniformly at the bottom of a 6-well plate using a marker pen. Cells in each group were treated with $0.25 \%$ trypsin, triturated into a single cell suspension, and counted. After that, the cells were seeded in a 6well plate at a density of $1 \times 10^{6}$ cells/well and mixed uniformly. After being cultured in a complete medium for 24 hours, the medium was replaced with RPMI 1640 medium containing $10 \%$ fetal bovine serum. Then, a $10 \mu \mathrm{L}$ sterile micropipette tip was used to make scratches perpendicular to the horizontal lines. Subsequently, caducous cells injured by micropipette tip were discarded. Serum-free medium and cells were cultured in a $5 \% \mathrm{CO}_{2}$ incubator at $37^{\circ} \mathrm{C}$. Cells were then imaged and observed at the 0 - and 24-hour time intervals under a microscope (Olympus). ${ }^{19}$

\section{Transwell Assay}

Matrigel (Sigma-Aldrich) was melted at $4^{\circ} \mathrm{C}$ overnight and diluted with $4^{\circ} \mathrm{C}$ precooled serum-free RPMI 1640 medium to a final concentration of $1 \mathrm{mg} / \mathrm{mL}$ on ice. Next, the diluted Matrigel $(80 \mu \mathrm{L} /$ well $)$ was vertically added to the basal central part in apical chamber of a transwell chamber (pore diameter, $8 \mu \mathrm{L})$. With uniformly distributed Matrigel, cells were incubated at $37^{\circ} \mathrm{C}$ for 4 hours. After cell detachment, cells were rinsed and suspended with serum-free RPMI 1640 medium and counted, with concentration adjusted to $1 \times 10^{6}$ cells $/ \mathrm{mL}$. RPMI 1640 medium $(700 \mu \mathrm{L})$ containing $10 \%$ fetal bovine serum was added to the basolateral chamber (the basal part of the 24-well plate), whereas the cell suspension was added to the apical chamber for a 24-hour incubation. Cells were then fixed with $4 \%$ paraformaldehyde at room temperature for 30 minutes and stained with $0.05 \%$ crystal violet at room temperature for 30 minutes. Cells in the apical chamber were removed with wet cotton swabs carefully and air dried. A total of 10 visual fields $(\times 200)$ were then randomly selected for cell counting under an inverted microscope (XSP-8CA; Shanghai Optical Instrument Factory, Shanghai, China).

\section{Xenograft Tumor in Nude Mice}

A total of 40 male BALB/c nude mice (aged 5 weeks, weighing 18 to $22 \mathrm{~g}$; Shanghai Experimental Animal Center of the Chinese Academy of Sciences, Shanghai, China) were 


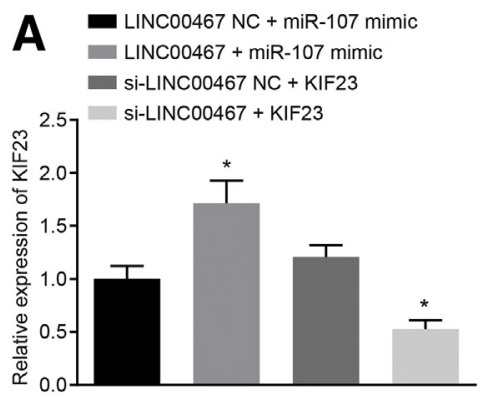

D $\begin{aligned} & \text { LINC00467 NC } \\ & + \text { miR-107 mimic }\end{aligned}$
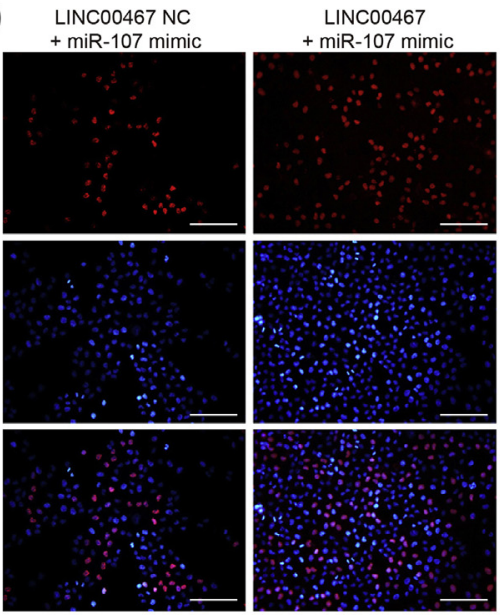

$\mathbf{F}$

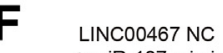
+ miR-107 mimic
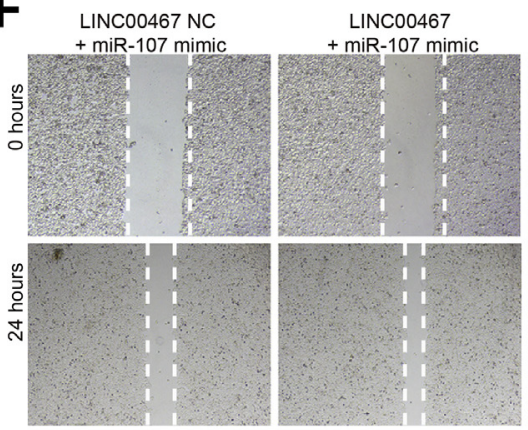

LINC00467 NC
+ miR-107 mimic LINC00467 + miR-107 mimic
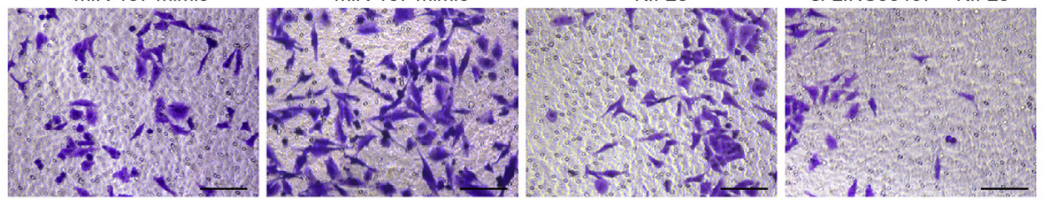

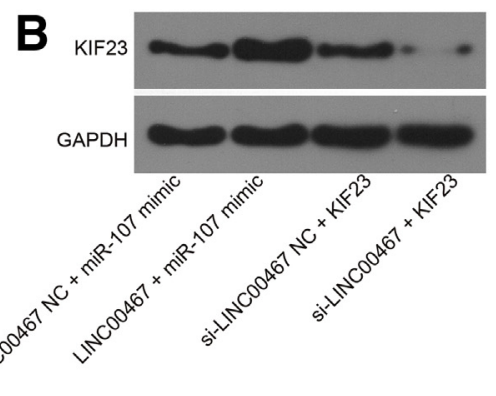

si-LINC00467 NC
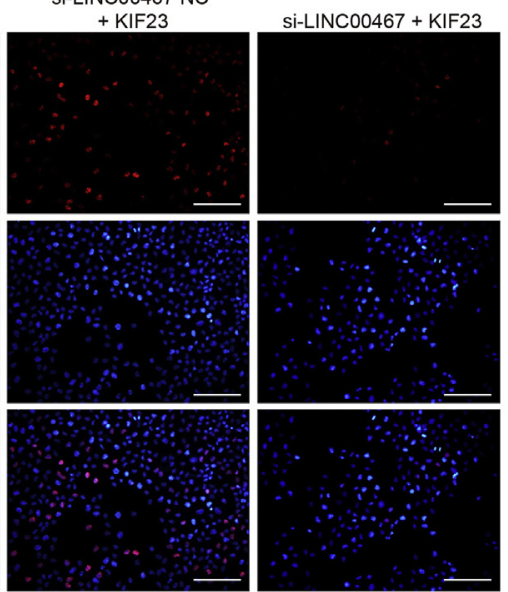

Si-LINC00467 NC

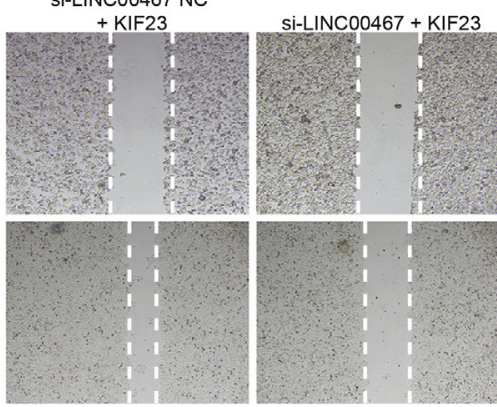

Si-LINC00467 NC $+\mathrm{KIF} 23$

si-LINC00467 + KIF23

\section{J LINC00467 NC + miR-107 mimic - LINC00467 + miR-107 mimic si-LINC00467 NC + KIF23}

$\left.{ }^{2.5}\right\rceil$ Si-LINC00467 + KIF23

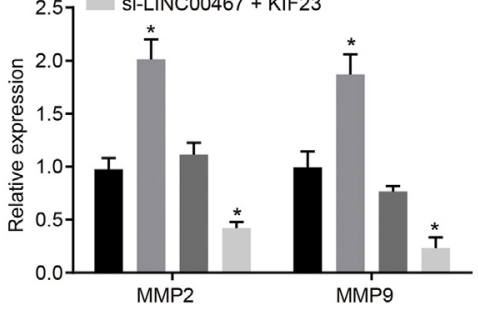

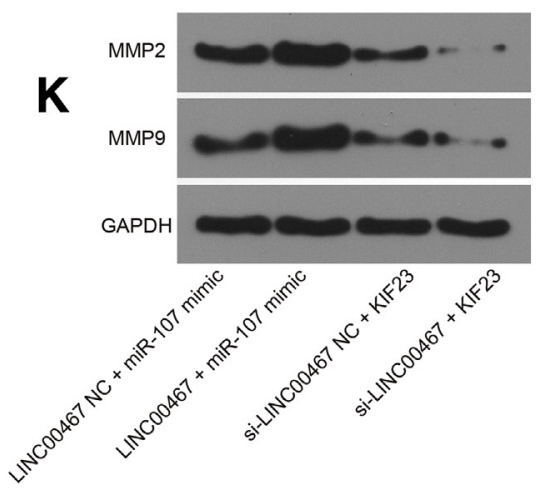

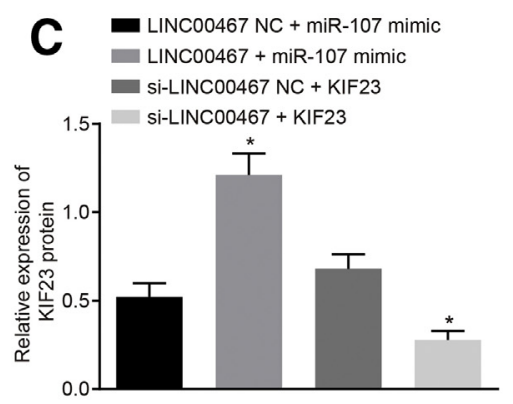

E

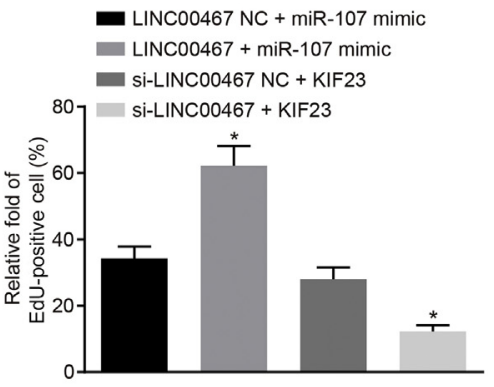

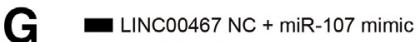 - LINC00467 + miR-107 mimic si-LINC00467 NC + KIF23
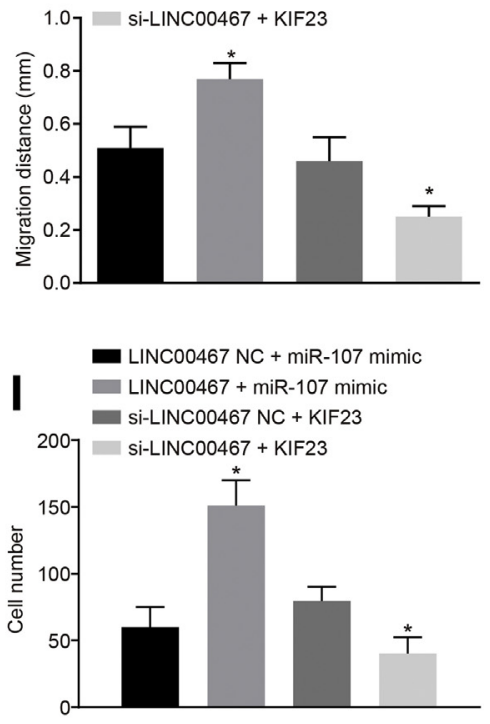

L LINC00467 NC + miR-107 mimic LINC00467 + miR-107 mimic si-LINC00467 NC + KIF23

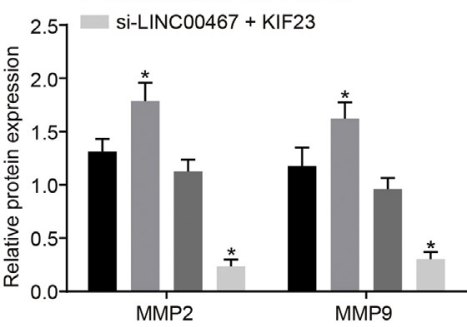


A
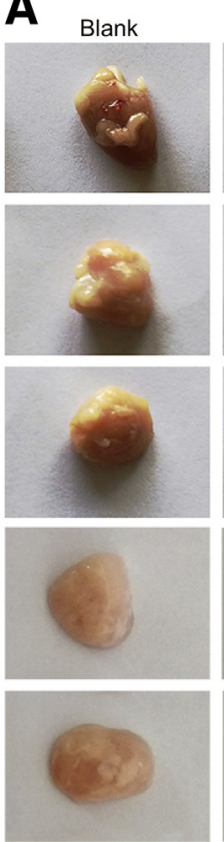

B
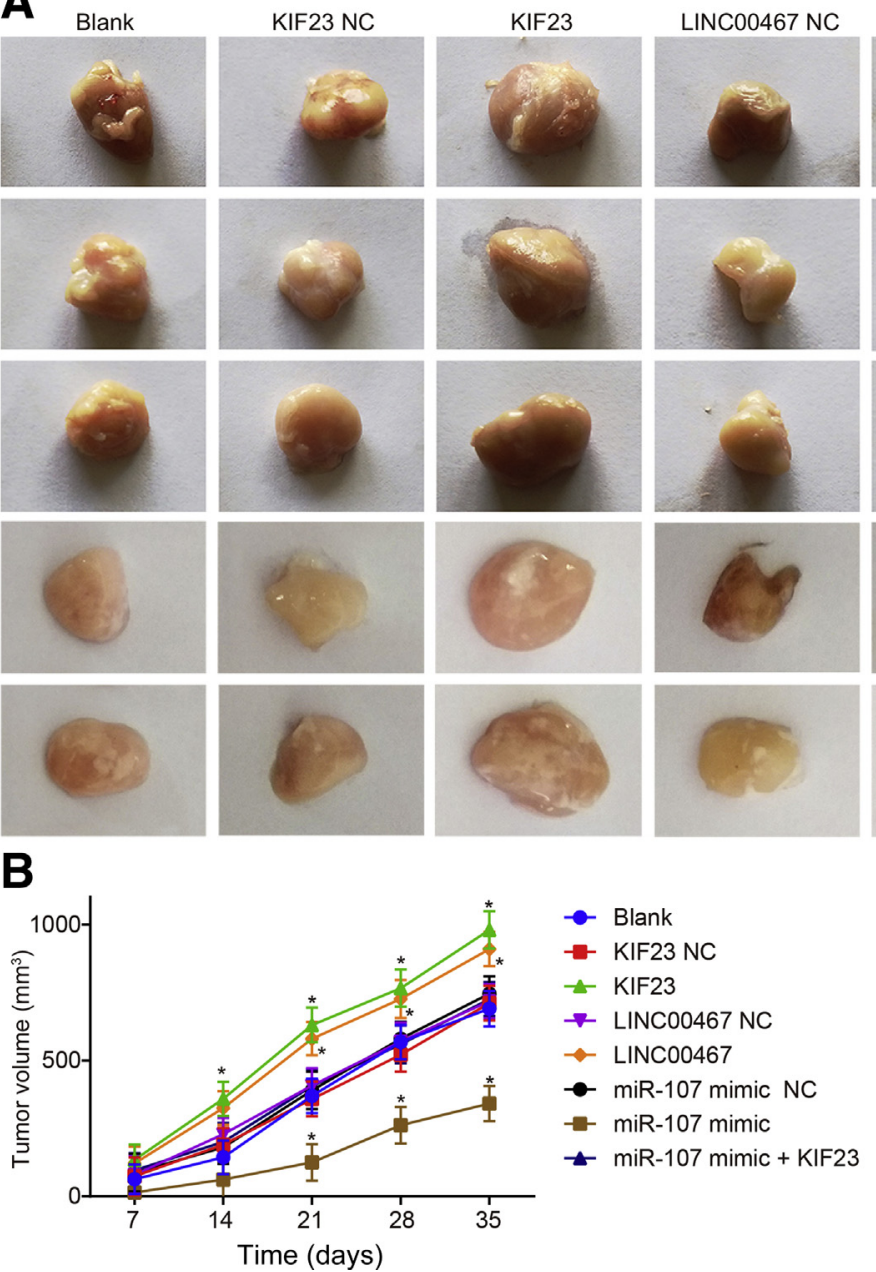
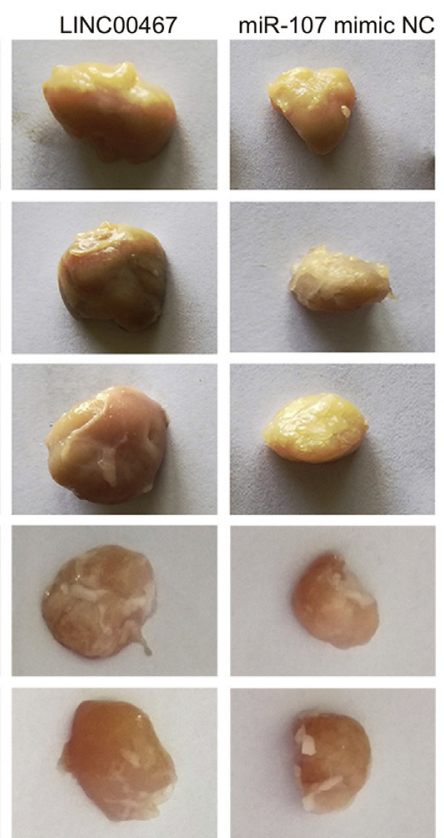

C
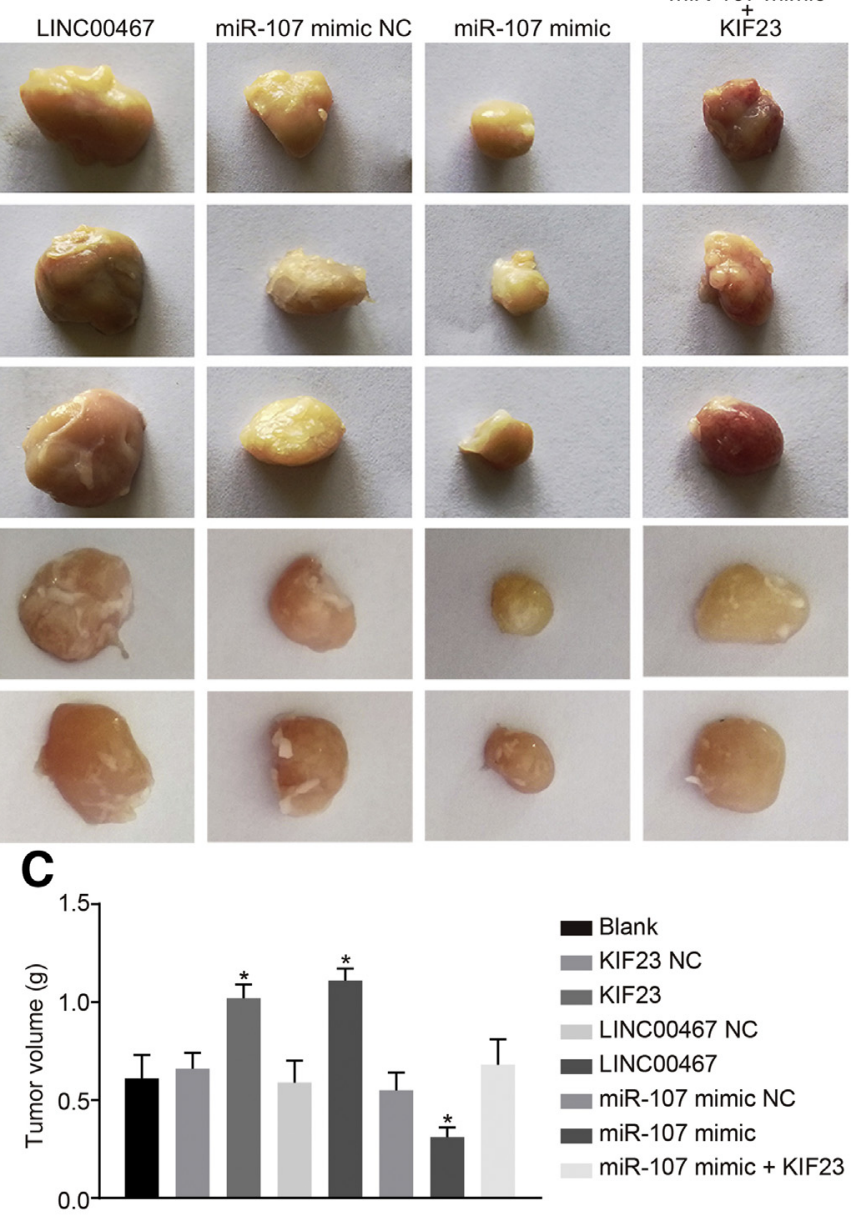

miR-107 mimic KIF̈23
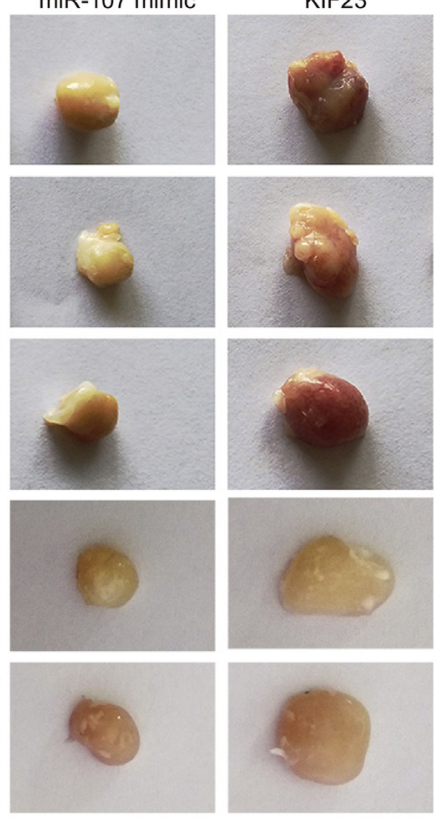

Figure 8 Silencing of LINC00467 or elevated miR-107 inhibits tumorigenic ability in nude mice in cervical cancer. A: Images showing xenograft tumor of nude mice. B: Tumor volume in nude mice after different treatment. C: Tumor weight in nude mice after different treatment. The volumes of xenograft tumor were measurement data, and tumor volume at different time points was analyzed by repeated measurement analysis of variance. Data are expressed as means \pm SD (B and $\mathbf{C}) . n=5$ (B and $\mathbf{C}$ ). ${ }^{*} P<0.05$ versus the blank group. KIF23, kinesin family member 23; NC, negative control.

randomly allocated into the following eight groups $(n=5$ per group): blank, KIF23, KIF23 NC, LINC00467, LINC00467 NC, miR-107 mimic, miR-107 mimic NC, and miR-107 mimic + KIF23 group. A stable transfected cell line was injected into the right axilla of nude mice. The nude mice were housed in a specific pathogen-free laboratory at constant temperature $\left(25^{\circ} \mathrm{C}\right)$ with humidity $(45 \%$ to $50 \%)$ in a 12 -hour dark/light cycle and were allowed free access to standard sterilized laboratory feed and water. After successful inoculation, tumor formation in nude mice was observed at the 7th, 14th, 21st, 28th, and 35th day. The major axis (a) and minor axis (b) of tumor were measured and recorded using a Vernier caliper. The volume of tumor $=\left(a \times b^{2}\right) / 2$. The growth curve of the tumor was plotted. Subsequently, the mice were euthanized using cervical dislocation on the 35th day. The skin was stripped to remove the complete transplanted tumor. An electronic balance was used to record the weight of the tumor. All experiments were performed in accordance with

Figure 7 Kinesin family member 23 (KIF23) expression is mediated by miR-107-dependent LINC00467 in cervical cancer. A: Graph showing mRNA expression of KIF23 in cervical cancer cells after transfection. B: Representative KIF23 protein band in cervical cancer cells after transfection. C: Graphs showing relative expression of KIF23 in cervical cancer cells after transfection. D: Representative images of cervical cancer cell proliferation detected by 5 ethynyl-2'-deoxyuridine (EdU) assay. E: Percentage of EdU-stained positive cervical cancer cell. F: Representative images showing the width of scratch in cervical cancer scratch test; the dotted lines indicate the current migration location of cells. G: Migration distance in cervical cancer cells after different treatment. H: Representative images showing cell invasion in transwell assay. I: Graph showing cervical cancer cell number in transwell assay. J: Graph showing relative expression patterns of matrix metallopeptidase (MMP) 2 and MMP9. K: Gray value analysis of MMP2, MMP9, and glyceraldehyde-3-phosphate dehydrogenase (GAPDH). L: Protein expression of MMP2 and MMP9. Immunofluorescence assay, cell proliferation value of each group in EdU assay, and migration distance in scratch test were measurement data, which were analyzed by one-way analysis of variance. The experiment was independently repeated three times. Data are expressed as means $\pm \mathrm{SD}(\mathbf{A}, \mathbf{C}, \mathbf{E}, \mathbf{G}, \mathbf{I}, \mathbf{J}$, and $\mathbf{L}) .{ }^{*} P<0.05$ versus the LINC00467 negative control (NC) + miR-107 mimic or siLINC00467 NC + KIF23 groups. Scale bars $=50 \mu \mathrm{m}(\mathbf{D}$ and $\mathbf{H})$. 

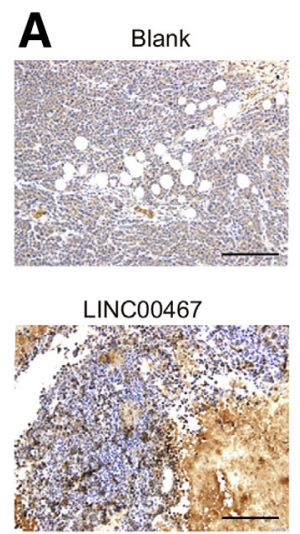

KIF23 NC

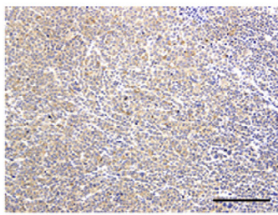

miR-107 mimic NC

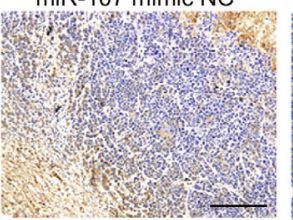

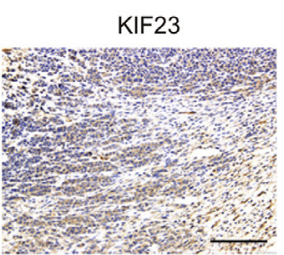

miR-107 mimic

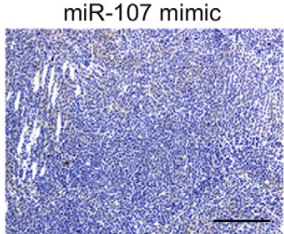

LINC00467 NC

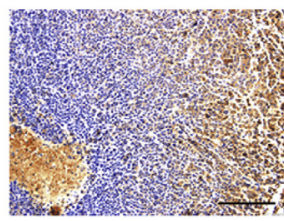

miR-107 mimic + KIF23

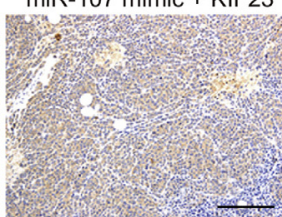

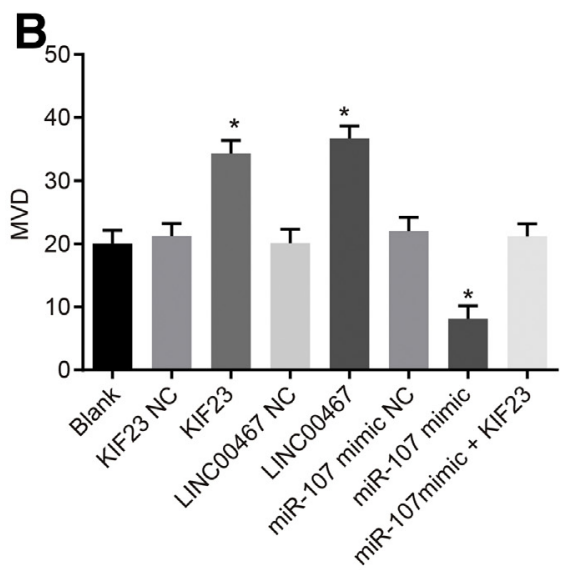

C

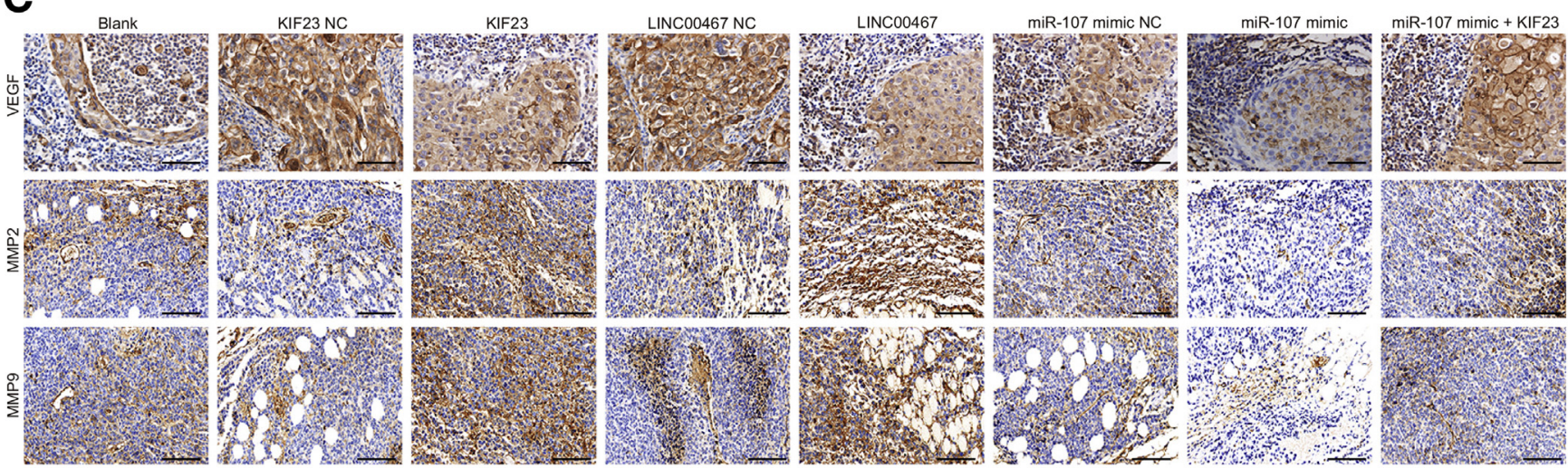

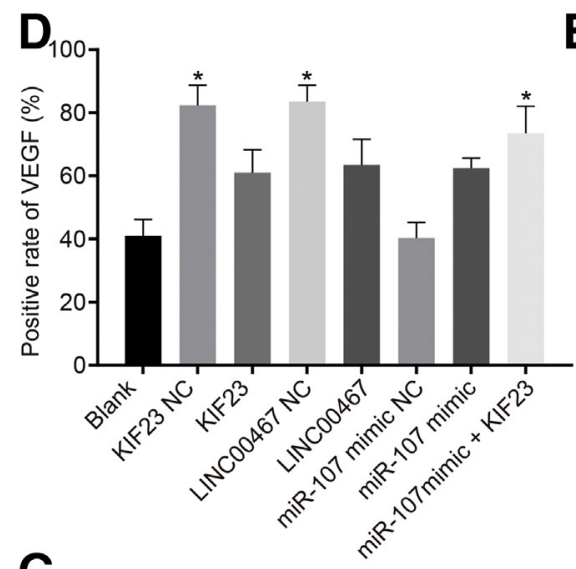

G
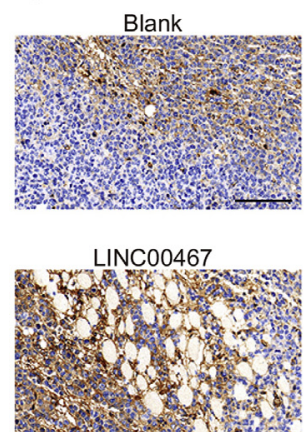

KIF23 NC

miR-107 mimic NC
E
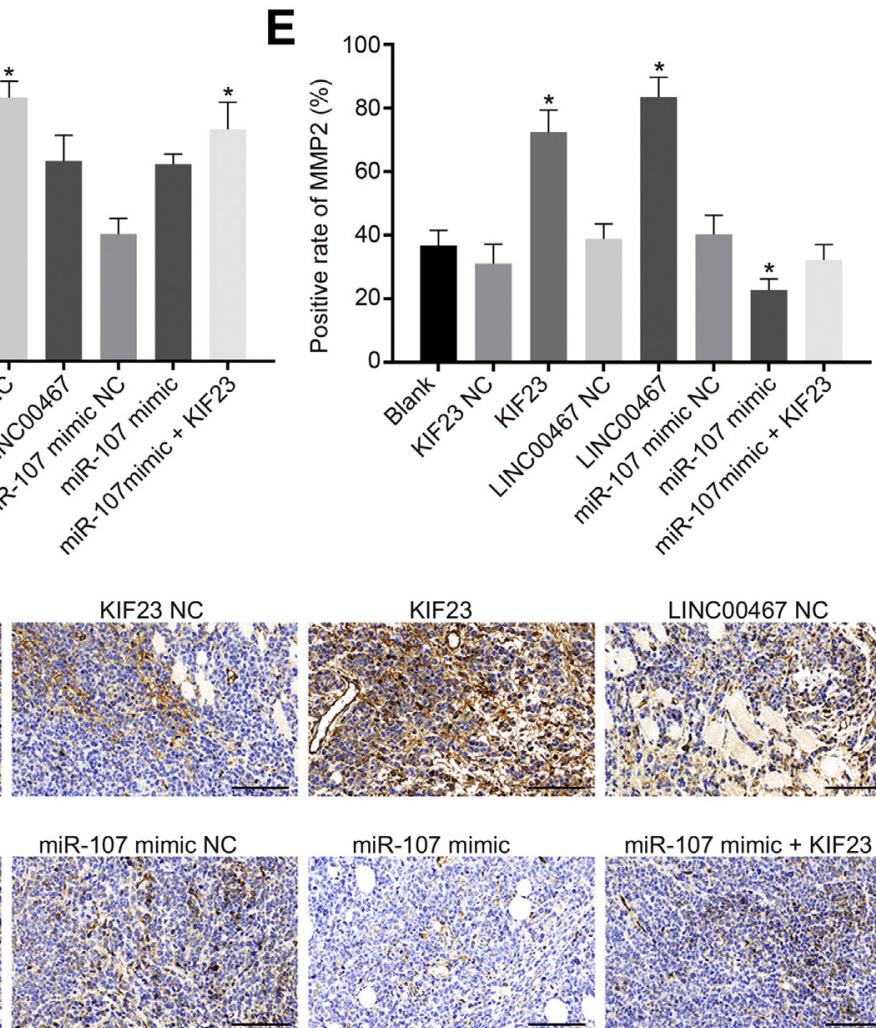
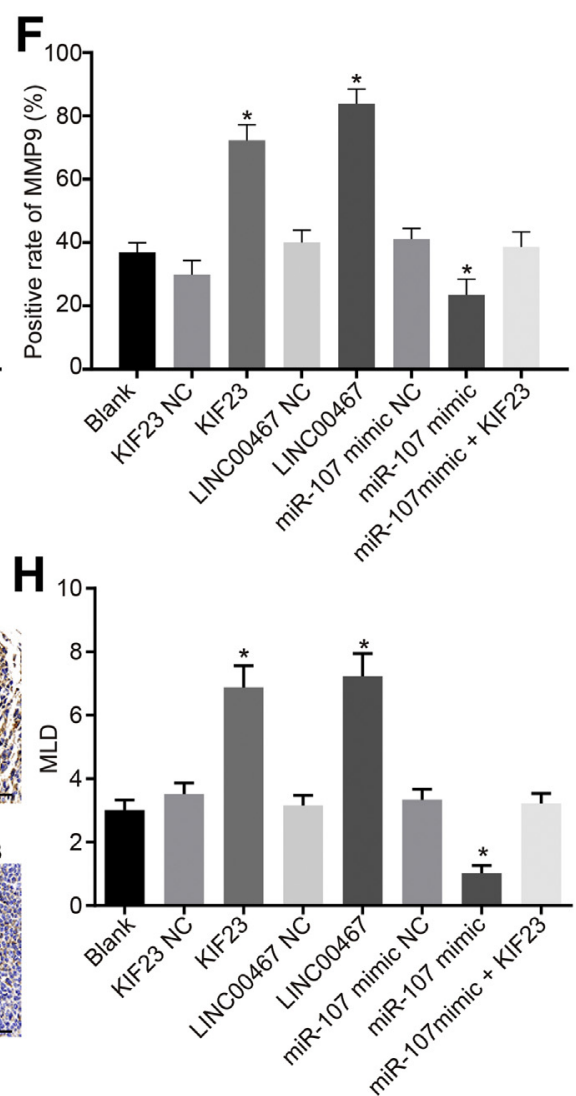
the Convention of International Laboratory Animal Ethics and complied with relevant national regulations.

\section{Immunohistochemistry}

The streptavidin-peroxidase method was used for staining the obtained tumor tissues. The tumor tissues in nude mice were fixed with $4 \%$ paraformaldehyde for 12 hours, dehydrated, cleared, embedded in paraffin, and then cut into slices. The thickness of immunohistochemical staining slices was $5 \mu \mathrm{m}$ and that of fluorescence in situ hybridization slices was $4 \mu \mathrm{m}$. Next, the slices were dewaxed in liquid, treated with antigen retrieval for 10 minutes, and added with $3 \% \mathrm{H}_{2} \mathrm{O}_{2}$. Slices were then sealed with sealing solution (5\% bovine serum albumin/ tris-buffered saline and Tween-20) for 15 minutes and incubated with the primary antibodies, rabbit anti-human antibodies to CD34 (ab81289; dilution 1:100), vascular endothelial growth factor (VEGF; ab32152; dilution 1:250), lymphatic vessel endothelial hyaluronan receptor-1 (ab14917; dilution 1:200), MMP2 (ab14917; dilution 1:200), and MMP9 (ab228402; dilution 1:200) at room temperature for 4 hours. After that, the slices were incubated with the secondary antibody, donkey anti-rabbit antibody to $\operatorname{IgG}$ (ab150073; dilution 1:200 to $1: 1000)$ at room temperature for 15 minutes. All aforementioned antibodies were purchased from Abcam (Cambridge, MA). Slices were then reacted with horseradish peroxidase-labeled streptavidin for 15 minutes and developed with diaminobenzidine. Next, the slices were counterstained with hematoxylin, followed by standard dehydration and vitrification, and sealed with neutral balsam. According to the Weidner counting method, the results were observed and recorded using a microscope (Olympus BX43; Olympus).

\section{Statistical Analysis}

Statistical analyses were performed using the SPSS software version 19.0 (IBM Corp., Armonk, NY). Overall survival and disease-free survival curves were plotted using the Kaplan-Meier method. Each experiment was independently repeated three times. Measurement data were presented as means \pm SD. Statistical comparisons between two groups with normal distribution were analyzed using the unpaired $t$-test. Multiple groups were compared by one-way analysis of variance. Normal distribution in multiple groups was analyzed by KolmogorovSmirnov method, and Tukey was used for post-hoc test. Repeated-measure analysis of variance was used to compare tumor volume at different time points. $P<0.05$ was statistically significant.

\section{Results}

LINC00467 and KIF23 Express at High Levels, whereas miR-107 Expresses at Low Levels, in Cervical Cancer

Microarray-based analyses on cervical cancer-related microarrays GSE7803 and GSE9750 revealed that KIF23 was highly expressed in cervical cancer (Figure 1, A and B). The Starbase website (http://starbase.sysu.edu.cn/targetSite. $p h p$; last accessed December 19, 2018) speculated that miR107, miR-15a-5p, and miR-103a-3p might be the target miRNAs for KIF23. In addition, RAID version 2.0 website (http://www.rna-society.org/rapoptotic; last accessed December 19, 2018) further verified the existence of a binding site between miR-107 and LINC00467. Furthermore, the GSE63514 microarray revealed that the expression of LINC00467 in cervical cancer was significantly higher compared with adjacent normal tissues (Figure 1C). The results of RT-qPCR (Figure 1D) and Western blot analysis showed that the expression of LINC00467 and KIF23 was increased, whereas that of miR-107 was decreased, in cervical cancer in comparison with the adjacent normal tissues (Figure 1, E and F). This relationship between LINC00467 and the clinicopathologic features of cervical cancer patients was evaluated further. The results showed that the expression of LINC00467 was not associated with the age of the patients, but was significantly correlated with the tumor size, differentiation, and tumornode-metastasis stage (Table 2). At the same time, survival analyses were performed using The Cancer Genome Atlas cervical cancer data, which revealed that high expression of LINC00467 was associated with a poor prognosis (Figure 1, G and $\mathrm{H}$ ). Furthermore, it was observed that LINC00467 was positively related to the expression of KIF23 in tumor samples $(P=0.006 r=0.371)$ (Figure 1I). These results suggested that LINC00467 might play a crucial role in the progression of cervical cancer by regulating the KIF23 expression.

\section{LINC00467 Is Primarily Located in Cytoplasm}

Fluorescence in situ hybridization was adopted to examine the subcellular location of LINC00467. The results (Figure 2) showed that LINC00467 was primarily found in the cytoplasm, with only a small amount in the cell nucleus. The red part represented LINC00467, and the blue represented the cell nucleus, suggesting that LINC00467 was mainly located in the cytoplasm.

Figure 9 LINC00467 silencing or enhanced miR-107 suppresses microvessel density (MVD) and vascular endothelial growth factor expression (VEGF) in xenograft tumor-bearing nude mice. A: Representative images showing immunohistochemistry of MVD. B: Graph showing MVD in xenograft tumor. C: Representative images showing immunohistochemistry of VEGF, matrix metallopeptidase (MMP) 2, and MMP9. D: Protein expression patterns of VEGF. E: Protein expression of MMP2. F: Graph showing relative expression of MMP9. G: Representative images showing immunohistochemistry of microvascular lymphatic density (MLD). H: Graph showing MLD in xenograft. Measurement data were analyzed by one-way analysis of variance. The experiment was independently repeated three times. Data are expressed as means \pm SD (B, D-F, and $\mathbf{H}) .{ }^{*} P<0.05$ versus the blank group. Scale bars: $50 \mu \mathrm{m}(\mathbf{A}$ and $\mathbf{G}) ; 25$ $\mu \mathrm{m}$ (C). KIF23, kinesin family member 23; NC, negative control. 


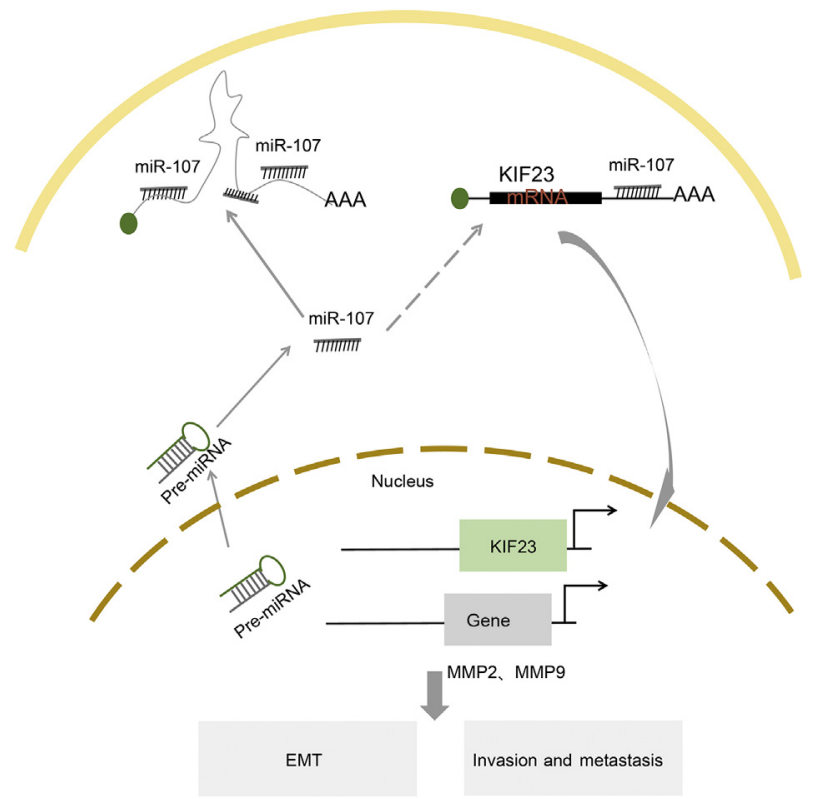

Figure 10 Schematic diagram showing LINC00467 acts as a competing endogenous RNA. LINC00467 sponges miR-107 in cervical cancer cells to reduce the inhibition of kinesin family member 23 (KIF23) and promote KIF23 expression, thus promoting epithelial-mesenchymal transition (EMT), proliferation, invasion, and metastasis in cervical cancer cells. MMP, matrix metallopeptidase.

Silencing of LINC00467 Represses EMT, Proliferation, Migration, and Invasion in Cervical Cancer Cells

To explore the mechanisms by which LINC00467 affected the tumorigenesis of cervical cancer, immunofluorescence assay was performed to measure the expression patterns of EMT-related proteins (Figure 3, A and B). Compared with the blank group, there were no significant differences in the expression of EMT-related proteins (N-cadherin and E-cadherin) in the si-LINC00467 NC and LINC00467 NC groups $(P>0.05)$. The expression of E-cadherin in the si-LINC00467 group was found to be significantly elevated, whereas that of $\mathrm{N}$-cadherin was significantly reduced, when compared with the blank group $(P<0.05)$. However, in the LINC00467 group, the expression of E-cadherin was downregulated, whereas $\mathrm{N}$-cadherin was increased, relative to the blank group $(P<0.05)$. In addition, cervical cancer cell proliferation was detected by means of EdU assay (Figure 3, C and D). Compared with the blank group, there was no significant difference between the si-LINC00467 NC and LINC00467 NC groups $(P>0.05)$. In contrast to the blank group, the number of EdU-positive cells was decreased significantly in the si-LINC00467 group $(P<0.05)$. EdU-positive cells were significantly increased in the LINC00467 group when compared with the blank group $(P<0.05)$. Furthermore, cell invasion and migration were examined by scratch test and transwell assay (Figure 3, E-K). Compared with the blank group, there were no differences in cell migration and invasion abilities between the
si-LINC00467 NC and LINC00467 NC groups $(P>0.05)$. In comparison to the blank group, cell migration and invasion were found to be significantly decreased, and the expression of MMP2 and MMP9 was significantly reduced, in the siLINC00467 group (all $P<0.05$ ). Cell migration, cell invasion, and MMP2 and MMP9 expression were noted to be significantly increased in the LINC00467 group when compared with the blank group (all $P<0.05$ ). Subsequently, the cervical cancer cell line SiHa was selected to detect the ability of cell proliferation, migration, and invasion; and the results were consistent with those of the HeLa cell line (Supplemental Figure S1). These results showed that silencing of LINC00467 repressed EMT, proliferation, migration, and invasion in cervical cancer cells.

\section{LINC00467 Sponges miR-107 to Regulate KIF23 by Functioning as a ceRNA}

To determine the targeting relationship between LINC00467 and miR-107, and between miR-107 and KIF23, bioinformatics tools and dual-luciferase reporter gene assay were used. The online prediction webites TargetScan and LncTBD revealed that there were binding sites between LINC00467 and miR-107 (Figure 4A), and between the $3^{\prime}$ untranslated region in KIF23 and miR-107 (Figure 4B), suggesting that miR-107 could bind to LINC00467 and KIF23 independently. Moreover, results from dualluciferase reporter gene assay showed that luciferase activity was significantly diminished in the miR-107 mimic group versus the NC group in LINC00467-WT plasmids $(P<0.05)$. However, there were no significant changes in the LINC00467-MUT plasmid $(P>0.05)$. Compared with the NC group, luciferase activity of miR-107 mimic group was found to be significantly decreased when transfected with KIF23-WT plasmids $(P<0.05)$, whereas there were no differences in the KIF23-MUT plasmids $(P>0.05)$. The aforementioned findings revealed the targeting relationship between LINC00467 and miR-107, as well as between miR107 and KIF23 (Figure 4, C and D). Furthermore, the results of RIP and RNA pull-down assays (Figure 4, E and F) showed that WT-miR-107 enriched more LINC00467 compared with MUT-miR-107 and bio-NC $(P<0.05)$, which further validated that LINC00467 binds to miR-107.

Next, specific experiments were designed to explore the relationship between LINC00467, miR-107, and KIF23 (Figure 4, G-I). Compared with the blank group, there were no significant differences in the expression of LINC00467, miR107, and KIF23 among the si-LINC00467 NC, LINC00467 NC, and LINC00467 MUT groups (all $P>0.05$ ). In the siLINC00467 group, the expression of LINC00467 and KIF23 was found to be significantly decreased, whereas that of miR107 was significantly increased, when compared with the blank group (all $P<0.05$ ). However, LINC00467 and KIF23 expression was increased, whereas the miR-107 expression was decreased, in the LINC00467 group relative to the blank group 
(both $P<0.05$ ). Thus, the above results indicated that LINC00467 competitively binds to miR-107 to regulate KIF23.

\section{Overexpressed miR-107 Inhibits Cervical Cancer Cell EMT, Proliferation, Migration, and Invasion}

To explore the role of miR-107 on tumor progression in cervical cancer, EdU assay, scratch test, and transwell assay were conducted. Compared with the blank group, there were no differences in the expression of E-cadherin and N-cadherin among the miR-107 mimic NC, KIF23 NC, and miR107 mimic + KIF23 groups (all $P>0.05$ ) (Figure 5, A and B). Although the expression of E-cadherin was found to be significantly elevated, that of $\mathrm{N}$-cadherin was significantly down-regulated, in the miR-107 mimic group when compared with the blank group (both $P<0.05$ ). The expression of E-cadherin was down-regulated, whereas that of N-cadherin was elevated, in the KIF23 group when compared with the blank group (both $P<0.05$ ). Results of the EdU assay showed that there were no differences in cell proliferation among the blank, miR-107 mimic NC, KIF23 NC, and miR-107 mimic + KIF23 groups (all $P>0.05$ ) (Figure 5, C and D). Relative to the blank group, EdUpositive cells were found to be significantly decreased in the miR-107 mimic group, suggesting that cell proliferation was repressed $(P<0.05)$. However, EdU-positive cells were significantly elevated in the KIF23 group versus the blank group, suggesting that cell proliferation was increased in the KIF23 group $(P<0.05)$. Furthermore, scratch test and transwell assay showed that, compared with the blank group, there were no differences in migration and invasion of cervical cancer cells among the miR-107 mimic NC, KIF23 NC, and miR-107 mimic + KIF23 groups (all $P>0.05$ ) (Figure 5, E-K). Cell migration and invasion in cervical cancer cells and the expression of metastasiscorrelated genes $(M M P 2$ and $M M P 9)$ were decreased in the miR-107 mimic group (all $P<0.05$ ). However, cell migration and invasion and expression of MMP2 and MMP9 were significantly increased in the KIF23 group (all $P<0.05$ ). Meanwhile, the above-mentioned experiments were repeated in the cervical cancer cell line $\mathrm{SiHa}$, and the results were the same as those obtained from the HeLa cell line (Supplemental Figure S2). Therefore, these results showed that overexpression of miR-107 inhibited cervical cancer cell EMT, proliferation, migration, and invasion.

Silencing of LINC00467 or Overexpression of miR-107 Inhibits KIF23

The expression of KIF23, LINC00467, and miR-107 after transfection was determined by RT-qPCR and Western blot analysis (Figure 6). Compared with the blank group, there were no significant differences in the expression of LINC00467, miR-107, and KIF23 among the miR-107 mimic NC, KIF23 NC, and miR-107 mimic + KIF23 groups (all
$P>0.05)$. In contrast to the blank group, the expression of LINC00467 and KIF23 was found to be evidently decreased, whereas the expression of miR-107 was significantly increased, in the miR-107 mimic group (all $P<0.05$ ). The expression of KIF23 was significantly increased in the KIF23 group compared with the blank group (all $P<0.05$ ). The results showed that silencing of LINC00467 or overexpression of miR-107 down-regulated KIF23.

\section{LINC00467 Competitively Sponges miR-107 to Modulate KIF23 Expression in Cervical Cancer}

To clarify the regulation of LINC00467 on downstream miR-107 and KIF23, LINC00467 NC + miR-107 mimic, LINC00467 + miR-107 mimic, si-LINC00467 $\mathrm{NC}+\mathrm{KIF} 23$, and si-LINC00467 + KIF23 groups were established. RT-qPCR and Western blot analysis were used to evaluate the expression of KIF23 after transfection. The results showed that the expression of KIF23 after LINC00467 + miR-107 mimic treatment was significantly higher than that in LINC00467 NC + miR-107 mimictransfected cells. At the same time, compared with the siLINC00467 NC + KIF23 treatment, siLINC00467 + KIF23 treatment significantly reduced expression of KIF23 (Figure 7). In addition, the proliferation, migration, and invasion abilities of cells were continually assessed in each group, and the results showed that, compared with the LINC00467 NC + miR-107 mimic group, LINC00467 treatment in the presence of miR-107 mimic could reverse the miR-107 mimic-induced cell proliferation, migration, and invasion abilities. However, when si-LINC00467 was cotransfected with KIF23, the cell proliferation, migration, and invasion abilities were found to be significantly decreased relative to si-LINC00467 NC plus KIF23 cotransfection (Figure 7). The results further demonstrated that LINC00467 could competitively sponge miR-107 to regulate the expression of KIF23.

Silencing of LINC00467 or Overexpression of miR-107 Represses Tumorigenic Ability in Nude Mice in Cervical Cancer

Xenograft tumor models were used in nude mice to determine changes in tumorigenic ability (Figure 8). Tumor volume and weight were not different among the KIF23 NC, LINC00467 NC, miR-107 mimic NC, and KIF23 + miR107 mimic groups when compared with the blank group (all $P>0.05$ ). Compared with the blank group, tumor volume was found to be significantly increased on the 7th, 14th, 21st, 28th, and 35th day after s.c. injection of transfected cells in the KIF23 and LINC00467 groups. Moreover, the weight of tumor was increased after the 35th day in the KIF23 and LINC00467 groups when compared with the blank group (all $P<0.05$ ). In the miR-107 mimic group, tumor volume was found to be significantly decreased on 
the 7th, 14th, 21st, 28th, and 35th day after s.c. injection of transfected cells. The weight of tumor was also decreased after the 35th day in the miR-107 mimic group (all $P<0.05$ ). Therefore, these findings suggested that silencing of LINC00467 or overexpression of miR-107 repressed tumorigenic ability in cervical cancer-bearing nude mice.

Silencing of LINC00467 or Overexpression of miR-107 Inhibits MVD and MLD and Lowers VEGF and MMP2/9 Expression of Xenograft Tumor in Nude Mice

Immunohistochemistry was used to determine the effect of LINC00467 and miR-107 on microvessel density (MVD), microvascular lymphatic density (MLD), VEGF, and MMP2/ 9 expression in cervical cancer tissues (Figure 9). Compared with the blank group, there were no differences in MVD, MLD, VEGF, and MMP2/9 expression among the KIF23 NC, LINC00467 NC, miR-107 mimic NC, and LINC00467 + miR-107 mimic groups (all $P>0.05$ ). However, significantly elevated MVD, MLD, VEGF, and MMP2/9 expression levels in xenograft tumor were observed in the KIF23 and LINC00467 groups $(P<0.05)$, whereas reduced MVD, MLD, VEGF, and MMP2/9 expression levels were observed in the miR-107 group $(P<0.05)$. These data revealed that silencing of LINC00467 or overexpression of miR-107 inhibited MVD, MLD, VEGF, and MMP2/9 expression in xenograft tumor models of nude mice.

\section{Discussion}

Cervical cancer is the leading cause of cancer-related deaths among women in developing countries, with approximately 500,000 cases being diagnosed every year worldwide. ${ }^{23}$ Progression in the treatment of cervical cancer, such as radical hysterectomy, has been made during the past few years; however, cervical cancer patients still experience postoperative long-term complications and poor prognoses. ${ }^{24}$ Recent exploration on the functions of IncRNAs in cervical cancer has demonstrated that several lncRNAs, including taurine up-regulated 1 and homeobox transcript antisense RNA, contribute to cervical cancer tumorigenesis. ${ }^{25,26}$ In the current study, we aimed to determine the effect of LINC00467 on the development of cervical cancer. Collectively, the data obtained from present study showed that silencing of LINC00467 down-regulates the expression of KIF23 by acting as a ceRNA against miR-107. Data further revealed that silencing of LINC00467 inhibits EMT, invasion, and migration in cervical cancer cells.

On the basis of the findings from dual-luciferase reporter gene assay and RIP and RNA pull-down assays, it was uncovered that LINC00467 competitively binds to miR-107 to regulate KIF23. In addition, the expression of LINC00467 and KIF23 was increased in cervical cancer tissues, whereas that of miR-107 was reduced. Our results were in agreement with a previous study that demonstrated that another lncRNA, cervical carcinoma high-expressed 1, presented with increased expression levels in cervical cancer tissues. ${ }^{27}$ Furthermore, LINC00467 has been previously found to be overexpressed in neuroblastoma cells, and its knockdown led to diminished neuroblastoma cell survival. ${ }^{9}$ Moreover, decreased expression of miR-107 is also known to correlate to poor prognosis in cervical cancer patients. ${ }^{12}$ In addition, another lncRNA, LINC00152, was previously demonstrated to promote glioblastoma progression by targeting miR-107. ${ }^{28}$ Similarly, Gius et al ${ }^{29}$ showed that KIF23 was overexpressed in normal cervical epithelium obtained from cervical cancer patients. Consistent with our findings, another recent study predicted that KIF23 was a target gene of miR-195. ${ }^{30}$ These findings demonstrate that LINC00467 serves as an oncogene to sponge miR-107 and regulate KIF23 in cervical cancer progression.

In addition, this study revealed that silencing of LINC00467 or overexpression of miR-107 elevated the expression of E-cadherin while reducing that of N-cadherin, MMP2, MMP9, VEGF, MLD, and MVD in cervical cancer in nude mice. E-cadherin and $\mathrm{N}$-cadherin are well known to be vital for normal cell adhesion, homeostasis, and tissue integrity. Recently, $\mathrm{Li}$ et al ${ }^{31}$ reported that elevation of E-cadherin relieves tumor burden and suppresses migration of cervical cancer cells. MMPs are related with HPV infections in cervical cancer patients, among which MMP2 and MMP9 are associated with poor prognoses in patients with invasive cervical cancer, and their knockdown decreases migration of cervical cancer cells. ${ }^{32}$ VEGF is known to play important roles in neoangiogenesis during disease progression and metastasis, and it is involved in the progression of cervical cancer. ${ }^{33}$ Furthermore, Saijo et $\mathrm{al}^{34}$ reported that increased MVD and VEGF expression was associated with unsatisfactory cervical cancer prognoses. Thus, these aforementioned studies affirm our findings that LINC00467 knockdown or miR-107 overexpression may play a role in the regulation of cervical cancer cell proliferation and tumorigenesis through the means of MMP2, MMP9, and VEGF.

Furthermore, the current study evidenced that silencing of LINC00467 or overexpression of miR-107 inhibits EMT, proliferation, migration, and invasion in cervical cancer by hindering the expression of KIF23. Likewise, several IncRNAs have been verified to play a central role in the proliferation and invasion of cervical cancer cells. ${ }^{35}$ For instance, inhibition of IncRNA plasmacytoma variant translocation 1 decreased cell proliferation, migration, and invasion, but increased apoptosis, in cervical cancer. ${ }^{36}$ Consistently, another study reported that overexpressed miR-107 acts as a tumor suppressor to inhibit cervical cancer cell invasion by negatively targeting myeloid cell leukemia $1 .{ }^{37}$ Also, Xiong et $\mathrm{al}^{38}$ demonstrated that deregulated miR-107 repressed cell invasion, migration, and EMT in pancreatic ductal adenocarcinoma. Furthermore, knockdown of KIF23 has been demonstrated to repress proliferation in glioma cells. ${ }^{39}$ The aforementioned results 
are in accordance with our results that silenced LINC00467 or overexpressed miR-107 restrains EMT, proliferation, migration, and invasion in cervical cancer by inhibiting KIF23.

On the whole, the current study evidenced the crucial effects of LINC00467 on cervical cancer. Moreover, this study endorsed the conception that LINC00467 mediates KIF23 by functioning as a ceRNA against miR-107 in cervical cancer cell EMT, invasion, and migration (Figure 10). These findings suggest that the LINC00467-miR-107-KIF23 axis might serve as an alternative therapeutic strategy for treatment and diagnosis of cervical cancer. Elucidation of the biological mechanism underlying the role of LINC00467 in tumorigenesis requires further research.

\section{Acknowledgments}

We thank the reviewers for their helpful comments.

G.-C.L. designed the study; Y.-S.W. collected data; Y.C. performed statistical analysis and prepared the figures; L.X. wrote the article; and all authors revised the article and read and approved the final version.

\section{Supplemental Data}

Supplemental material for this article can be found at http://doi.org/10.1016/j.ajpath.2019.07.012.

\section{References}

1. Siva S, Deb S, Young RJ, Hicks RJ, Callahan J, Bressel M, Mileshkin L, Rischin D, Bernshaw D, Narayan K: (1)(8)F-FDG $\mathrm{PET} / \mathrm{CT}$ following chemoradiation of uterine cervix cancer provides powerful prognostic stratification independent of HPV status: a prospective cohort of 105 women with mature survival data. Eur J Nucl Med Mol Imaging 2015, 42:1825-1832

2. Hillmann Ede C, Dos Reis R, Monego H, Appel M, Hammes LS, Rivoire WA, Capp E: Cervical digital photography for screening of uterine cervix cancer and its precursor lesions in developing countries. Arch Gynecol Obstet 2013, 288:183-189

3. Sherman SM, Lane EL: Awareness of risk factors for breast, lung and cervical cancer in a UK student population. J Cancer Educ 2015, 30: 660-663

4. Moon JY, Song IC, Ko YB, Lee HJ: The combination of cisplatin and topotecan as a second-line treatment for patients with advanced/recurrent uterine cervix cancer. Medicine 2018, 97:e0340

5. Seo Y, Kim MS, Yoo HJ, Jang WI, Rhu SY, Choi SC, Kim MH, Kim BJ, Lee DH, Cho CK: Salvage stereotactic body radiotherapy for locally recurrent uterine cervix cancer at the pelvic sidewall: feasibility and complication. Asia Pac J Clin Oncol 2016, 12:e280-e288

6. Cao W, Peng T, Zhou Y: Long noncoding RNA activated by transforming growth factor-beta promotes cancer development and is a prognostic marker in cervical cancer. J Cancer Res Ther 2017, 13:801-806

7. Kim HJ, Eoh KJ, Kim LK, Nam EJ, Yoon SO, Kim KH, Lee JK, Kim SW, Kim YT: The long noncoding RNA HOXA11 antisense induces tumor progression and stemness maintenance in cervical cancer. Oncotarget 2016, 7:83001-83016

8. Yang L, Yi K, Wang H, Zhao Y, Xi M: Comprehensive analysis of lncRNAs microarray profile and mRNA-lncRNA co-expression in oncogenic HPV-positive cervical cancer cell lines. Oncotarget 2016, 7:49917-49929

9. Atmadibrata B, Liu PY, Sokolowski N, Zhang L, Wong M, Tee AE, Marshall GM, Liu T: The novel long noncoding RNA linc00467 promotes cell survival but is down-regulated by N-Myc. PLoS One 2014, 9:e88112

10. Park HJ, Ji P, Kim S, Xia Z, Rodriguez B, Li L, Su J, Chen K, Masamha CP, Baillat D, Fontes-Garfias CR, Shyu AB, Neilson JR, Wagner EJ, Li W: 3' UTR shortening represses tumor-suppressor genes in trans by disrupting ceRNA crosstalk. Nat Genet 2018, 50: 783-789

11. Chen J, Zhou X, Xiao Q, Wang T, Shao G, Li Y, Zhang Z: MiR-107 suppresses cell proliferation and tube formation of Ewing sarcoma cells partly by targeting HIF-1beta. Hum Cell 2018, 31:42-49

12. Dong P, Xiong Y, Hanley SJB, Yue J, Watari H: Musashi-2, a novel oncoprotein promoting cervical cancer cell growth and invasion, is negatively regulated by 553 -induced miR-143 and miR-107 activation. J Exp Clin Cancer Res 2017, 36:150

13. Sun L, Zhang C, Yang Z, Wu Y, Wang H, Bao Z, Jiang T: KIF23 is an independent prognostic biomarker in glioma, transcriptionally regulated by TCF-4. Oncotarget 2016, 7:24646-24655

14. Sun $X$, Jin Z, Song X, Wang J, Li Y, Qian X, zhang Y, Yin Y: Evaluation of KIF23 variant 1 expression and relevance as a novel prognostic factor in patients with hepatocellular carcinoma. BMC Cancer 2015, 15:961

15. Zou JX, Duan Z, Wang J, Sokolov A, Xu J, Chen CZ, Li JJ, Chen HW: Kinesin family deregulation coordinated by bromodomain protein ANCCA and histone methyltransferase MLL for breast cancer cell growth, survival, and tamoxifen resistance. Mol Cancer Res 2014, 12:539-549

16. Gautier L, Cope L, Bolstad BM, Irizarry RA: affy: analysis of Affymetrix GeneChip data at the probe level. Bioinformatics 2004, 20:307-315

17. Smyth GK: Linear models and empirical bayes methods for assessing differential expression in microarray experiments. Stat Appl Genet Mol Biol 2004, 3. Article3

18. Hale RW: XIX FIGO World Congress of Gynecology and Obstetrics. Int J Gynaecol Obstet 2009, 106:189-190

19. Alexopoulou AN, Leao M, Caballero OL, Da Silva L, Reid L, Lakhani SR, Simpson AJ, Marshall JF, Neville AM, Jat PS: Dissecting the transcriptional networks underlying breast cancer: NR4A1 reduces the migration of normal and breast cancer cell lines. Breast Cancer Res 2010, 12:R51

20. Collin SP: Topographic organization of the ganglion cell layer and intraocular vascularization in the retinae of two reef teleosts. Vision Res 1989, 29:765-775

21. Luan W, Zhou Z, Ni X, Xia Y, Wang J, Yan Y, Xu B: Long noncoding RNA H19 promotes glucose metabolism and cell growth in malignant melanoma via miR-106a-5p/E2F3 axis. J Cancer Res Clin Oncol 2018, 144:531-542

22. Guo T, Wang W, Zhang H, Liu Y, Chen P, Ma K, Zhou C: ISL1 promotes pancreatic islet cell proliferation. PLoS One 2011, 6:e22387

23. Mileshkin L, Paramanathan A, Kondalsamy-Chennakesavan S, Bernshaw D, Khaw P, Narayan K: Smokers with cervix cancer have more uterine corpus invasive disease and an increased risk of recurrence after treatment with chemoradiation. Int J Gynecol Cancer 2014, 24:1286-1291

24. Oh JK, Park NH, Oh SJ: Effect of the systematised critical pathway protocol on emptying failure as a secondary complication of radical hysterectomy due to uterine cervix cancer. J Clin Nurs 2014, 23:1702-1707

25. Hu Y, Sun X, Mao C, Guo G, Ye S, Xu J, Zou R, Chen J, Wang L, Duan P, Xue X: Upregulation of long noncoding RNA TUG1 promotes cervical cancer cell proliferation and migration. Cancer Med 2017, 6:471-482

26. Li Q, Feng Y, Chao X, Shi S, Liang M, Qiao Y, Wang B, Wang P, Zhu Z: HOTAIR contributes to cell proliferation and metastasis of cervical cancer via targetting miR-23b/MAPK1 axis. Biosci Rep 2018, 38. BSR20171563 
27. Mutimura M, Ebong C, Rao IM, Nsahlai IV: Nutritional values of available ruminant feed resources in smallholder dairy farms in Rwanda. Trop Anim Health Prod 2015, 47:1131-1137

28. Liu X, Yidayitula Y, Zhao H, Luo Y, Ma X, Xu M: LncRNA LINC00152 promoted glioblastoma progression through targeting the miR-107 expression. Environ Sci Pollut Res Int 2018, 25:17674-17681

29. Gius D, Funk MC, Chuang EY, Feng S, Huettner PC, Nguyen L, Bradbury CM, Mishra M, Gao S, Buttin BM, Cohn DE, Powell MA, Horowitz NS, Whitcomb BP, Rader JS: Profiling microdissected epithelium and stroma to model genomic signatures for cervical carcinogenesis accommodating for covariates. Cancer Res 2007, 67: $7113-7123$

30. Monkeviciute A: Analysis of microRNA role in the development of left ventricular hypertrophy in the stroke-prone spontaneously hypertensive rat. WHO AIDS Tech Bull 2014, 2:106-107

31. Li X, Wang H, Du X, Yu W, Jiang J, Geng Y, Guo X, Fan X, Ma C: Lactobacilli inhibit cervical cancer cell migration in vitro and reduce tumor burden in vivo through upregulation of E-cadherin. Oncol Rep 2017, 38:1561-1568

32. Zhu D, Ye M, Zhang W: E6/E7 oncoproteins of high risk HPV-16 upregulate MT1-MMP, MMP-2 and MMP-9 and promote the migration of cervical cancer cells. Int J Clin Exp Pathol 2015, 8:4981-4989

33. Kotowicz B, Fuksiewicz M, Jonska-Gmyrek J, Bidzinski M, Kowalska M: The assessment of the prognostic value of tumor markers and cytokines as SCCAg, CYFRA 21.1, IL-6, VEGF and
sTNF receptors in patients with squamous cell cervical cancer, particularly with early stage of the disease. Tumour Biol 2016, 37: $1271-1278$

34. Saijo Y, Furumoto H, Yoshida K, Nishimura M, Irahara M: Clinical significance of vascular endothelial growth factor expression and microvessel density in invasive cervical cancer. J Med Invest 2015, 62:154-160

35. Chen J, Fu Z, Ji C, Gu P, Xu P, Yu N, Kan Y, Wu X, Shen R, Shen Y: Systematic gene microarray analysis of the lncRNA expression profiles in human uterine cervix carcinoma. Biomed Pharmacother 2015, 72:83-90

36. Iden M, Fye S, Li K, Chowdhury T, Ramchandran R, Rader JS: The IncRNA PVT1 contributes to the cervical cancer phenotype and associates with poor patient prognosis. PLoS One 2016, 11: e0156274

37. Zhou C, Li G, Zhou J, Han N, Liu Z, Yin J: miR-107 activates ATR/Chk1 pathway and suppress cervical cancer invasion by targeting MCL1. PLoS One 2014, 9:e111860

38. Xiong J, Wang D, Wei A, Lu H, Tan C, Li A, Tang J, Wang Y, He S, Liu X, Hu W: Deregulated expression of miR-107 inhibits metastasis of PDAC through inhibition PI3K/Akt signaling via caveolin-1 and PTEN. Exp Cell Res 2017, 361:316-323

39. Takahashi S, Fusaki N, Ohta S, Iwahori Y, Iizuka Y, Inagawa K, Kawakami Y, Yoshida K, Toda M: Downregulation of KIF23 suppresses glioma proliferation. J Neurooncol 2012, 106:519-529 